EUROPEAN ORGANISATION FOR NUCLEAR RESEARCH

CERN-PPE/95-179

8th December 1995

(Revised 8th February 1996)

\title{
Measurement of Heavy Quark Forward-Backward Asymmetries and Average B Mixing Using Leptons in Multihadronic Events
}

\author{
The OPAL Collaboration
}

\begin{abstract}
A measurement of the forward-backward asymmetries of $\mathrm{e}^{+} \mathrm{e}^{-} \rightarrow \mathrm{Z}^{0} \rightarrow \mathrm{b} \overline{\mathrm{b}}$ and $\mathrm{e}^{+} \mathrm{e}^{-} \rightarrow \mathrm{Z}^{0} \rightarrow c \bar{c}$ using electrons and muons produced in semileptonic decays of bottom and charm hadrons is presented. Two new variables designed to optimize flavour separation are used in a maximum likelihood fit to a sample of events containing one or two identified leptons, allowing the simultaneous extraction of the $b$ and c quark forward-backward asymmetries as well as the average $\mathrm{B}^{0}-\overline{\mathrm{B}^{0}}$ mixing. Using all data collected by OPAL up to the end of 1994 , the asymmetries are measured to be:

$$
\begin{aligned}
& A_{\mathrm{FB}}^{\mathrm{b}}=(5.5 \pm 2.4 \pm 0.3) \% \quad A_{\mathrm{FB}}^{\mathrm{c}}=(-7.5 \pm 3.4 \pm 0.6) \% \quad \text { at }\langle\sqrt{s}\rangle=89.52 \mathrm{GeV}, \\
& A_{\mathrm{FB}}^{\mathrm{b}}=(9.06 \pm 0.51 \pm 0.23) \% \quad A_{\mathrm{FB}}^{\mathrm{c}}=(6.00 \pm 0.67 \pm 0.52) \% \quad \text { at }\langle\sqrt{s}\rangle=91.24 \mathrm{GeV}, \\
& A_{\mathrm{FB}}^{\mathrm{b}}=(11.7 \pm 2.0 \pm 0.3) \% \quad A_{\mathrm{FB}}^{\mathrm{c}}=(14.1 \pm 2.8 \pm 0.9) \% \quad \text { at }\langle\sqrt{s}\rangle=92.94 \mathrm{GeV} \text {. }
\end{aligned}
$$

For the average $\mathrm{B}^{0}-\overline{\mathrm{B}^{0}}$ mixing a value of:

$$
\bar{\chi}=0.1107 \pm 0.0062 \pm 0.0055,
$$

is obtained, where in each case the first error is statistical and the second systematic.

(To be submitted to Zeitschrift für Physik C) 


\section{The OPAL Collaboration}

G. Alexander ${ }^{23}$, J. Allison ${ }^{16}$, N. Altekamp ${ }^{5}$, K. Ametewee ${ }^{25}$, K.J. Anderson ${ }^{9}$, S. Anderson ${ }^{12}$, S. Arcelli ${ }^{2}$, S. Asai ${ }^{24}$, D. Axen ${ }^{29}$, G. Azuelos ${ }^{18, a}$, A.H. Ball ${ }^{17}$, E. Barberio ${ }^{26}$, R.J. Barlow ${ }^{16}$, R. Bartoldus ${ }^{3}$, J.R. Batley ${ }^{5}$, G. Beaudoin ${ }^{18}$, J. Bechtluft ${ }^{14}$, G.A. Beck ${ }^{13}$, C. Beeston ${ }^{16}$, T. Behnke ${ }^{8}$, A.N.Bell ${ }^{1}$, K.W.Bell ${ }^{20}$, G. Bella ${ }^{23}$, S. Bentvelsen ${ }^{8}$, P. Berlich ${ }^{10}$, S. Bethke ${ }^{14}$, O. Biebel ${ }^{14}$, I.J. Bloodworth ${ }^{1}$, J.E. Bloomer ${ }^{1}$, P. Bock ${ }^{11}$, H.M. Bosch ${ }^{11}$, M. Boutemeur ${ }^{18}$, B.T. Bouwens ${ }^{12}$, S. Braibant ${ }^{12}$, P. Bright-Thomas ${ }^{25}$, R.M. Brown ${ }^{20}$, H.J. Burckhart ${ }^{8}$, C. Burgard ${ }^{27}$, R. Bürgin ${ }^{10}$, P. Capiluppi ${ }^{2}$, R.K.Carnegie ${ }^{6}$, A.A.Carter ${ }^{13}$, J.R. Carter ${ }^{5}$, C.Y.Chang ${ }^{17}$, C. Charlesworth ${ }^{6}$, D.G. Charlton ${ }^{1, b}$, D. Chrisman ${ }^{4}$, S.L.Chu ${ }^{4}$, P.E.L. Clarke ${ }^{15}$, S.G. Clowes ${ }^{16}$, I. Cohen $^{23}$, J.E. Conboy ${ }^{15}$, O.C.Cooke ${ }^{16}$, M. Cuffiani ${ }^{2}$, S. Dado ${ }^{22}$, C. Dallapiccola ${ }^{17}$, G.M.Dallavalle ${ }^{2}$, C. Darling ${ }^{31}$, S. De Jong ${ }^{12}$, L.A. del Pozo ${ }^{8}$, M.S. Dixit ${ }^{7}$, E. do Couto e Silva ${ }^{12}$, E. Duchovni ${ }^{26}$, G. Duckeck ${ }^{8}$, I.P. Duerdoth ${ }^{16}$, U.C. Dunwoody ${ }^{8}$, J.E.G. Edwards ${ }^{16}$, P.G. Estabrooks ${ }^{6}$, H.G. Evans ${ }^{9}$, F. Fabbri ${ }^{2}$, B. Fabbro ${ }^{21}$, P. Fath ${ }^{11}$, F. Fiedler ${ }^{12}$, M. Fierro ${ }^{2}$, M. Fincke-Keeler ${ }^{28}$, H.M. Fischer ${ }^{3}$, R. Folman ${ }^{26}$, D.G. Fong ${ }^{17}$,

M. Foucher ${ }^{17}$, H. Fukui ${ }^{24}$, A. Fürtjes ${ }^{8}$, P. Gagnon ${ }^{7}$, A. Gaidot ${ }^{21}$, J.W. Gary ${ }^{4}$, J. Gascon ${ }^{18}$,

S.M. Gascon-Shotkin ${ }^{17}$, N.I. Geddes ${ }^{20}$, C. Geich-Gimbel ${ }^{3}$, S.W.Gensler ${ }^{9}$, F.X. Gentit ${ }^{21}$, T. Geralis ${ }^{20}$, G. Giacomelli ${ }^{2}$, P. Giacomelli ${ }^{4}$, R. Giacomelli ${ }^{2}$, V. Gibson ${ }^{5}$, W.R. Gibson ${ }^{13}$, D.M. Gingrich ${ }^{30, a}$, J.Goldberg ${ }^{22}$, M.J.Goodrick ${ }^{5}$, W. Gorn ${ }^{4}$, C. Grandi ${ }^{2}$, E. Gross ${ }^{26}$, C. Hajdu ${ }^{32}$, G.G. Hanson ${ }^{12}$, M.Hansroul ${ }^{8}$, M.Hapke ${ }^{13}$, C.K. Hargrove ${ }^{7}$, P.A. Hart ${ }^{9}$, C.Hartmann ${ }^{3}$, M. Hauschild ${ }^{8}$, C.M.Hawkes ${ }^{8}$,

R. Hawkings ${ }^{8}$, R.J. Hemingway ${ }^{6}$, G. Herten ${ }^{10}$, R.D. Heuer ${ }^{8}$, M.D. Hildreth ${ }^{8}$, J.C. Hill ${ }^{5}$, S.J. Hillier ${ }^{8}$, T. Hilse ${ }^{10}$, P.R. Hobson ${ }^{25}$, D. Hochman ${ }^{26}$, R.J. Homer ${ }^{1}$, A.K.Honma ${ }^{28, a}$, D. Horváth ${ }^{32, c}$, R. Howard ${ }^{29}$,

R.E. Hughes-Jones ${ }^{16}$, D.E. Hutchcroft ${ }^{5}$, P. Igo-Kemenes ${ }^{11}$, D.C.Imrie ${ }^{25}$, A. Jawahery ${ }^{17}$,

P.W. Jeffreys ${ }^{20}$, H. Jeremie ${ }^{18}$, M. Jimack ${ }^{1}$, A. Joly ${ }^{18}$, M. Jones ${ }^{6}$, R.W.L. Jones ${ }^{8}$, U. Jost ${ }^{11}$, P. Jovanovic $^{1}$, D. Karlen ${ }^{6}$, T. Kawamoto ${ }^{24}$, R.K. Keeler ${ }^{28}$, R.G. Kellogg ${ }^{17}$, B.W. Kennedy ${ }^{20}$, B.J. King ${ }^{8}$, J. King ${ }^{13}$, J. Kirk ${ }^{29}$, S. Kluth ${ }^{5}$, T. Kobayashi ${ }^{24}$, M. Kobel ${ }^{10}$, D.S. Koetke ${ }^{6}$, T.P. Kokott ${ }^{3}$,

S. Komamiya ${ }^{24}$, R. Kowalewski ${ }^{8}$, T. Kress ${ }^{11}$, P. Krieger ${ }^{6}$, J. von Krogh ${ }^{11}$, P. Kyberd ${ }^{13}$,

G.D. Lafferty ${ }^{16}$, H. Lafoux $^{21}$, R. Lahmann ${ }^{17}$, W.P. Lai ${ }^{19}$, D. Lanske ${ }^{14}$, J. Lauber ${ }^{15}$, J.G. Layter ${ }^{4}$,

A.M. Lee ${ }^{31}$, E. Lefebvre ${ }^{18}$, D. Lellouch ${ }^{26}$, J. Letts ${ }^{2}$, L. Levinson ${ }^{26}$, C. Lewis ${ }^{15}$, S.L. Lloyd ${ }^{13}$,

F.K. Loebinger ${ }^{16}$, G.D. Long17, B. Lorazo ${ }^{18}$, M.J. Losty ${ }^{7}$, J. Ludwig ${ }^{10}$, A. Luig10, A. Malik²1, M. Mannelli ${ }^{8}$, S. Marcellini ${ }^{2}$, C. Markus ${ }^{3}$, A.J. Martin ${ }^{13}$, J.P. Martin ${ }^{18}$, G. Martinez ${ }^{17}$, T. Mashimo ${ }^{24}$, W. Matthews ${ }^{25}$, P. Mättig ${ }^{3}$, W.J. McDonald ${ }^{30}$, J. McKenna ${ }^{29}$, E.A. Mckigney ${ }^{15}$, T.J. McMahon ${ }^{1}$, A.I. McNab ${ }^{13}$, F. Meijers ${ }^{8}$, S. Menke ${ }^{3}$, F.S.Merritt ${ }^{9}$, H. Mes ${ }^{7}$, J. Meyer ${ }^{27}$, A. Michelini ${ }^{8}$, G. Mikenberg ${ }^{26}$, D.J. Miller ${ }^{15}$, R. Mir $^{26}$, W. Mohr ${ }^{10}$, A. Montanari ${ }^{2}$, T. Mori ${ }^{24}$, M. Morii ${ }^{24}$, U. Müller ${ }^{3}$,

B. Nellen ${ }^{3}$, B. Nijjhar ${ }^{16}$, R. Nisius ${ }^{8}$, S.W. O'Neale ${ }^{1}$, F.G. Oakham ${ }^{7}$, F. Odorici ${ }^{2}$, H.O. Ogren ${ }^{12}$, N.J. Oldershaw ${ }^{16}$, T. Omori ${ }^{24}$, C.J. Oram ${ }^{28, a}$, M.J. Oreglia ${ }^{9}$, S. Orito ${ }^{24}$, M.Palazzo ${ }^{2}$, J.Pálinkás ${ }^{33}$, F.M.Palmonari ${ }^{2}$, J.P. Pansart ${ }^{21}$, G. ásztor $^{32}$, J.R.Pater ${ }^{16}$, G.N.Patrick ${ }^{20}$, M.J. Pearce ${ }^{1}$, P.D. Phillips ${ }^{16}$, J.E. Pilcher ${ }^{9}$, J. Pinfold ${ }^{30}$, D.E.Plane ${ }^{8}$, P. Poffenberger ${ }^{28}$, B. Poli ${ }^{2}$, A. Posthaus ${ }^{3}$, T.W.Pritchard ${ }^{13}$, H. Przysiezniak ${ }^{30}$, D.L. Rees ${ }^{1}$, D. Rigby ${ }^{1}$, M.G. Rison ${ }^{5}$, S.A. Robins ${ }^{13}$, N. Rodning ${ }^{30}$, J.M. Roney ${ }^{28}$, E. Ros $^{8}$, A.M. Rossi ${ }^{2}$, M. Rosvick $^{28}$, P. Routenburg ${ }^{30}$, Y. Rozen ${ }^{8}$, K. Runge ${ }^{10}$, O. Runolfsson ${ }^{8}$, D.R. Rust ${ }^{12}$, R. Rylko ${ }^{25}$, E.K.G.Sarkisyan ${ }^{23}$, M.Sasaki ${ }^{24}$, C.Sbarra ${ }^{2}$, A.D.Schaile ${ }^{8}$, O.Schaile ${ }^{10}$, F.Scharf ${ }^{3}$, P.Scharff-Hansen ${ }^{8}$, P. Schenk ${ }^{4}$, B. Schmitt ${ }^{3}$, M.Schröder ${ }^{8}$, H.C.Schultz-Coulon ${ }^{10}$, M.Schulz ${ }^{8}$, P.Schütz ${ }^{3}$, J.Schwiening ${ }^{3}$, W.G.Scott ${ }^{20}$, T.G.Shears ${ }^{16}$, B.C.Shen ${ }^{4}$, C.H.Shepherd-Themistocleous ${ }^{27}$, P.Sherwood ${ }^{15}$, G.P.Siroli ${ }^{2}$, A.Sittler ${ }^{27}$, A.Skillman ${ }^{15}$, A.Skuja ${ }^{17}$, A.M.Smith ${ }^{8}$, T.J.Smith ${ }^{28}$, G.A.Snow ${ }^{17}$, R. Sobie ${ }^{28}$, S.Söldner-Rembold ${ }^{10}$,

R.W.Springer ${ }^{30}$, M.Sproston ${ }^{20}$, A.Stahl ${ }^{3}$, M.Starks ${ }^{12}$, C.Stegmann ${ }^{10}$, K. Stephens ${ }^{16}$, J.Steuerer ${ }^{28}$, B. Stockhausen ${ }^{3}$, D. Strom ${ }^{19}$, F.Strumia ${ }^{8}$, P.Szymanski ${ }^{20}$, R. Tafirout ${ }^{18}$, H. Takeda ${ }^{24}$, P. Taras ${ }^{18}$, S. Tarem ${ }^{22}$, M. Tecchio ${ }^{8}$, N. Tesch ${ }^{3}$, M.A. Thomson ${ }^{8}$, E. von Törne ${ }^{3}$, S. Towers ${ }^{6}$, M. Tscheulin ${ }^{10}$, T.Tsukamoto ${ }^{24}$, E.Tsur ${ }^{23}$, A.S.Turcot ${ }^{9}$, M.F.Turner-Watson ${ }^{8}$, P. Utzat ${ }^{11}$, R. Van Kooten ${ }^{12}$, G. Vasseur ${ }^{21}$, P. Vikas ${ }^{18}$, M.Vincter ${ }^{28}$, E.H. Vokurka ${ }^{16}$, F. Wäckerle ${ }^{10}$, A. Wagner ${ }^{27}$, D.L. Wagner ${ }^{9}$, 


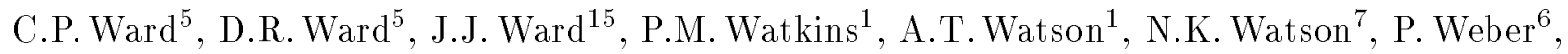
P.S. Wells ${ }^{8}$, N. Wermes ${ }^{3}$, B. Wilkens ${ }^{10}$, G.W.Wilson ${ }^{27}$, J.A. Wilson ${ }^{1}$, T. Wlodek ${ }^{26}$, G. Wolf ${ }^{26}$, S. Wotton ${ }^{11}$, T.R. Wyatt ${ }^{16}$, S. Xella ${ }^{2}$, S. Yamashita ${ }^{24}$, G. Yekutieli ${ }^{26}$, V. Zacek ${ }^{18}$,

${ }^{1}$ School of Physics and Space Research, University of Birmingham, Birmingham B15 2TT, UK

${ }^{2}$ Dipartimento di Fisica dell' Università di Bologna and INFN, I-40126 Bologna, Italy

${ }^{3}$ Physikalisches Institut, Universität Bonn, D-53115 Bonn, Germany

${ }^{4}$ Department of Physics, University of California, Riverside CA 92521, USA

${ }^{5}$ Cavendish Laboratory, Cambridge CB3 0HE, UK

${ }^{6}$ Ottawa-Carleton Institute for Physics, Department of Physics, Carleton University, Ottawa, Ontario K1S 5B6, Canada

${ }^{7}$ Centre for Research in Particle Physics, Carleton University, Ottawa, Ontario K1S 5B6, Canada

${ }^{8}$ CERN, European Organisation for Particle Physics, CH-1211 Geneva 23, Switzerland

${ }^{9}$ Enrico Fermi Institute and Department of Physics, University of Chicago, Chicago IL 60637, USA

${ }^{10}$ Fakultät für Physik, Albert Ludwigs Universität, D-79104 Freiburg, Germany

${ }^{11}$ Physikalisches Institut, Universität Heidelberg, D-69120 Heidelberg, Germany

${ }^{12}$ Indiana University, Department of Physics, Swain Hall West 117, Bloomington IN 47405, USA

${ }^{13}$ Queen Mary and Westfield College, University of London, London E1 4NS, UK

${ }^{14}$ Technische Hochschule Aachen, III Physikalisches Institut, Sommerfeldstrasse 26-28, D-52056 Aachen, Germany

${ }^{15}$ University College London, London WC1E 6BT, UK

${ }^{16}$ Department of Physics, Schuster Laboratory, The University, Manchester M13 9PL, UK

${ }^{17}$ Department of Physics, University of Maryland, College Park, MD 20742, USA

${ }^{18}$ Laboratoire de Physique Nucléaire, Université de Montréal, Montréal, Quebec H3C 3J7, Canada

${ }^{19}$ University of Oregon, Department of Physics, Eugene OR 97403, USA

${ }^{20}$ Rutherford Appleton Laboratory, Chilton, Didcot, Oxfordshire OX11 0QX, UK

${ }^{21}$ CEA, DAPNIA/SPP, CE-Saclay, F-91191 Gif-sur-Yvette, France

${ }^{22}$ Department of Physics, Technion-Israel Institute of Technology, Haifa 32000, Israel

${ }^{23}$ Department of Physics and Astronomy, Tel Aviv University, Tel Aviv 69978, Israel

${ }^{24}$ International Centre for Elementary Particle Physics and Department of Physics, University of

Tokyo, Tokyo 113, and Kobe University, Kobe 657, Japan

${ }^{25}$ Brunel University, Uxbridge, Middlesex UB8 3PH, UK

${ }^{26}$ Particle Physics Department, Weizmann Institute of Science, Rehovot 76100, Israel

${ }^{27}$ Universität Hamburg/DESY, II Institut für Experimental Physik, Notkestrasse 85, D-22607 Hamburg, Germany

${ }^{28}$ University of Victoria, Department of Physics, P O Box 3055, Victoria BC V8W 3P6, Canada

${ }^{29}$ University of British Columbia, Department of Physics, Vancouver BC V6T 1Z1, Canada

${ }^{30}$ University of Alberta, Department of Physics, Edmonton AB T6G 2J1, Canada

${ }^{31}$ Duke University, Dept of Physics, Durham, NC 27708-0305, USA

${ }^{32}$ Research Institute for Particle and Nuclear Physics, H-1525 Budapest, P O Box 49, Hungary

${ }^{33}$ Institute of Nuclear Research, H-4001 Debrecen, P O Box 51, Hungary

${ }^{a}$ Also at TRIUMF, Vancouver, Canada V6T $2 \mathrm{~A} 3$

${ }^{b}$ Royal Society University Research Fellow

${ }^{c}$ Institute of Nuclear Research, Debrecen, Hungary 


\section{Introduction}

The measurement of the forward-backward asymmetries of heavy quarks, $A_{\mathrm{FB}}^{\mathrm{q}}(\mathrm{q}=\mathrm{b}, \mathrm{c})$, provides an important test of the Standard Model. It allows the determination of $\sin ^{2} \theta_{\mathrm{W}}$ to high precision [1] and, in case of a deviation from the Standard Model, may constrain its extensions.

The forward-backward asymmetry of $\mathrm{e}^{+} \mathrm{e}^{-} \rightarrow Z^{0} \rightarrow \mathrm{q} \overline{\mathrm{q}}$ is induced by the parity violating nature of the neutral weak current, which manifests itself as an odd $\operatorname{contribution}$ in $\cos \theta$ in the differential cross-section:

$$
\frac{\mathrm{d} \sigma}{\mathrm{d} \cos \theta} \propto 1+\cos ^{2} \theta+\frac{8}{3} A_{\mathrm{FB}}^{\mathrm{q}} \cos \theta
$$

where $\theta$ denotes the angle between the outgoing quark and the incoming electron flight direction. The asymmetry $A_{\mathrm{FB}}^{\mathrm{q}}$ is related to the vector, $g_{V}$, and axial-vector, $g_{A}$, couplings of the $Z^{0}$ to the electron and quark q. At the $\mathrm{Z}^{0}$ resonance, it has the approximate form:

$$
A_{\mathrm{FB}}^{\mathrm{q}} \approx \frac{3}{4} \frac{2 g_{A \mathrm{e}} g_{V \mathrm{e}}}{g_{A \mathrm{e}}^{2}+g_{V \mathrm{e}}^{2}} \frac{2 g_{A \mathrm{q}} g_{V \mathrm{q}}}{g_{A \mathrm{q}}{ }^{2}+g_{V \mathrm{q}}{ }^{2}} .
$$

The asymmetry for $b \bar{b}$ events is diluted by the effect of mixing in the $\mathrm{B}^{0}-\overline{\mathrm{B}^{0}}$ system, which arises from box diagrams involving mainly virtual top quarks [2]. The average mixing parameter, $\bar{\chi}$, is the probability that a produced $b$ hadron decays as its antiparticle. It relates the observed $b$ quark asymmetry $A_{\mathrm{FB}}^{\mathrm{b}, o b s}$ to the theoretical asymmetry $A_{\mathrm{FB}}^{\mathrm{b}}$ through:

$$
A_{\mathrm{FB}}^{\mathrm{b}, \mathrm{obs}}=(1-2 \bar{\chi}) A_{\mathrm{FB}}^{\mathrm{b}} \text {. }
$$

OPAL and other LEP experiments [3,4], have already reported on several measurements of $A_{\mathrm{FB}}^{\mathrm{b}}$ and $A_{\mathrm{FB}}^{\mathrm{c}}$ making use of three distinct techniques: prompt leptons [5], fully reconstructed $\mathrm{D}^{*}$ mesons [6], and a jet-charge algorithm combined with a lifetime tag [7].

The measurement presented here uses leptons produced in semileptonic decays of $b$ and $c$ hadrons, usually referred to as 'prompt' leptons. These leptons are known to offer one of the most efficient means of tagging at the same time the flavour and the charge of the primary quark. Though similar in many aspects to the previous such measurement performed by OPAL [5], this analysis uses a nearly 10 times larger data sample and benefits from three important modifications:

- The electron identification algorithm is new, and was designed to optimize both the efficiency and the uniformity of the geometrical acceptance of the selection.

- The $\mathrm{B}^{0}-\overline{\mathrm{B}^{0}}$ mixing parameter is measured together with the asymmetries, by considering simultaneously events with one or two identified leptons. Fitting at the same time for asymmetries and mixing allows all correlations to be taken into account.

- Flavour separation between the different sources of prompt leptons has been significantly improved by the use of optimized variables. These variables, which combine jet-shape, lifetime and kinematical information are provided by neural network algorithms.

This paper is organized as follows. A brief description of the event selection and the simulated data sample used in this analysis is given in section 2. The new electron identification algorithm is presented in section 3, while section 4 summarizes the results for both lepton selections. Section 5 introduces the two new variables designed to improve flavour separation. The fitting method, results and systematic uncertainties are presented respectively in sections 6,7 and 8 . 


\section{Event Selection and Simulation}

\subsection{Event Selection}

This analysis is performed on all data collected by OPAL during five years of running, from 1990 to 1994. The OPAL detector has been described elsewhere [8]. It is equipped with a silicon microvertex detector, a vertex chamber, a jet chamber and z-chambers, positioned inside a solenoid that provides a uniform magnetic field of $0.435 \mathrm{~T}$. The coil is surrounded by a lead-glass electromagnetic calorimeter with a presampler, a hadron calorimeter and muon chambers.

The multihadronic event selection has been described in detail in a previous publication [9]. In addition, each event has to contain at least seven charged tracks satisfying minimal quality requirements. A total of about 3.6 million events are selected, $90 \%$ of which were recorded on the $Z^{0}$ peak (table 1$)$.

For each event, the thrust axis is calculated from both charged tracks and electromagnetic calorimeter energy clusters not associated to tracks. Only events with $\left|\cos \theta_{\text {thrust }}\right|<0.95$ are considered. Jets are reconstructed using a cone jet-finding algorithm [10] by combining all charged tracks and unassociated electromagnetic calorimeter energy clusters. This algorithm is preferred over the more standard JADE algorithm [11] for this measurement because it leads to a higher probability of correct assignment of tracks to secondary $b$ vertices within a jet.

Leptons are searched for in each jet using the algorithms described below. Only events where one lepton or two 'opposite-jet' leptons have been identified are used. For dilepton events, the two jets containing the leptons are considered as 'opposite' if their axes do not belong to the same thrust hemisphere. Table 1 summarizes the total number of hadronic $Z^{0}$ decays passing the multihadronic event selection as well as the number of events containing one candidate electron or muon or two opposite-jet leptons.

\begin{tabular}{|ll|r|r|r|r|}
\hline Mean Energy & $\begin{array}{c}\text { Total number } \\
\text { of } \mathrm{Z}^{0} \text { decays }\end{array}$ & $\begin{array}{c}\text { Single electron } \\
\text { events }\end{array}$ & $\begin{array}{c}\text { Single muon } \\
\text { events }\end{array}$ & $\begin{array}{c}\text { Dilepton } \\
\text { events }\end{array}$ \\
\hline 'peak-2' & $\langle\sqrt{s}\rangle=89.52 \mathrm{GeV}$ & $132 \mathrm{k}$ & 6383 & 6783 & 930 \\
'peak' & $\langle\sqrt{s}\rangle=91.24 \mathrm{GeV}$ & $3305 \mathrm{k}$ & 157069 & 172595 & 23553 \\
'peak+2' & $\langle\sqrt{s}\rangle=92.94 \mathrm{GeV}$ & $190 \mathrm{k}$ & 8837 & 10223 & 1386 \\
\hline
\end{tabular}

Table 1: Total number of multihadronic events and number of single and dilepton events remaining after the lepton selection. $\langle\sqrt{s}\rangle$ denotes the mean centre-of-mass energy for the selected events.

\subsection{Simulation}

Simulated data satisfying the same event and lepton selection criteria as the real data are used to predict the distributions of the variables over which the fit is performed. All the Monte Carlo samples were generated with the same version of the JETSET 7.3 program [12] whose parameters have been tuned to OPAL data [13], and passed through the detector simulation program [14], providing a total of 3 million fully simulated multihadronic events.

The fragmentation of heavy quarks is described in the simulation by the fragmentation function of Peterson et al. [15], with parameters $\epsilon_{\mathrm{b}}=0.0055$ and $\epsilon_{\mathrm{C}}=0.070$, corresponding to mean scaled energies of $\left\langle x_{E}\right\rangle_{\mathrm{b}}=0.70$ and $\left\langle x_{E}\right\rangle_{\mathrm{c}}=0.49$. The semileptonic decay model of Altarelli et al. [16] with parameters fixed by CLEO, DELCO and MARKIII data [17-19] is used to predict the lepton momentum in the rest frame of $\mathrm{b}$ and $\mathrm{c}$ hadrons. 


\section{Electron Identification Using a Neural Network}

\subsection{Description of the Algorithm}

The electron selection is based on a set of twelve physical quantities measured for each track in the central tracking chambers, the presampler and the lead-glass electromagnetic calorimeter:

- $p$, the track momentum;

- $\cos \theta$, the cosine of the polar angle of the track ${ }^{1}$;

- $\mathrm{d} E / \mathrm{d} x$, the specific ionization energy loss of the track in the central tracking chamber;

- $\sigma(\mathrm{d} E / \mathrm{d} x)$, the estimated error on $\mathrm{d} E / \mathrm{d} x$;

- $E / p$, the energy in the electromagnetic calorimeter cluster associated to the track divided by the track momentum;

- the total number of lead-glass blocks in the electromagnetic cluster;

- $E_{\text {cone }} / p$, where $E_{\text {cone }}$ is the sum of the energy deposited in the lead-glass blocks whose centre is contained within a cone of half angle $30 \mathrm{mrad}$ around the track direction;

- the number of lead glass blocks in the cone;

- $E_{\text {cone }} /\left(E_{\text {cone }}+\Delta E\right)$, where $\Delta E$ is the energy contained in all blocks adjacent to the blocks used to calculate $E_{\text {cone }}$;

- $\theta_{\text {track }}-\theta_{\text {cluster }}$, the difference in $\theta$ between the track position extrapolated to the electromagnetic calorimeter and the centre of the electromagnetic cluster;

- $\phi_{\text {track }}-\phi_{\text {cluster }}$, the difference in $\phi$ between the track position extrapolated to the electromagnetic calorimeter and the centre of the electromagnetic cluster;

- the multiplicity of the presampler cluster associated to the track.

The high level of complementarity and redundancy between these variables, provided by several independent subdetectors, ensures a good efficiency and rejection over the whole detector. A powerful multidimensional algorithm is needed in order to make optimal use of all the available information. These quantities are therefore used as input to a neural network [21] which provides the final classification.

The chosen set of inputs has been selected from a much larger set of potentially discriminating or meaningful variables. While most of those retained have intrinsic separating power between hadrons and electrons (such as $\mathrm{d} E / \mathrm{d} x, E / p, \ldots$ ), some variables which are not discriminating in themselves (e.g., the momentum $p$ and the polar angle $\cos \theta$ of the track) are nevertheless useful in that they contain important information about existing correlations between all inputs.

The network used is of the feed-forward type with one hidden layer made of 15 nodes. It was trained on simulated data to discriminate between electrons and hadrons, in the momentum range $p>2 \mathrm{GeV} / c$. At the end of this learning phase, the network's output can be computed for any track in real data events. This output, denoted $\mathrm{NET}_{\mathrm{el}}$, is a real function of the inputs whose value, ranging from 0 to 1 , is a measure of the probability that the track being considered is an electron.

\footnotetext{
${ }^{1}$ The OPAL coordinate system is cylindrical, with the $z$ axis along the $\mathrm{e}^{-}$beam direction and polar and azimuthal angle $\theta$ and $\phi$.
} 


\subsection{Performance}

This electron selection has been designed specifically for analyses where a high and smooth efficiency over the whole acceptance is needed, while a precise knowledge of its absolute value is not required. A first version of this algorithm has in fact already been used in several OPAL measurements, for instance in [20].

The simulation which is used to predict the efficiency and misidentification probability of the algorithm was checked in bins of momentum and polar angle. A study of a nearly pure sample of electrons obtained from photon conversions showed that in the momentum range $p>2 \mathrm{GeV} / c$, Monte Carlo estimates of efficiencies are reliable with an accuracy of $\pm 2.5 \%$ (figure 1 ).

A test sample of pions obtained by kinematical identification of $\mathrm{K}^{0} \rightarrow \pi^{+} \pi^{-}$decays also allowed a direct comparison of misidentification probabilities between data and Monte Carlo (figure 1). The Monte Carlo prediction was found to be reliable with a relative uncertainty of $\pm 15 \%$.

Figure 2 compares the $\mathrm{NET}_{\text {el }}$ distributions measured in the Monte Carlo and in the data for all tracks with momentum greater than $2 \mathrm{GeV} / c$. Sensible cut values on the output vary between 0.8 and 0.95. In figure 3 , the efficiency to identify prompt electrons in the same momentum range is plotted as a function of the polar angle $\theta$ of the track. The $|\cos \theta|$ distribution of all candidate electrons selected in the data by the cut $\mathrm{NET}_{\mathrm{el}}>0.95$ is also indicated.

\section{Lepton Selection}

\subsection{Electron selection}

Tracks with momentum greater than $2 \mathrm{GeV} / c$ are considered as electrons if their network output $\mathrm{NET}_{\text {el }}$ is greater than 0.95 (figure 2). This cut has been chosen so as to minimize the sensitivity to uncertainties in the background level while ensuring a high efficiency (approximately 71\%).

After the electron selection, electrons from photon conversion are the dominant background to prompt electrons. They are tagged and rejected using an algorithm that tries to associate any given candidate electron track with all possible partner tracks in the event. The decision whether a pair of tracks forms a vertex compatible with a photon conversion relies on the measurement of nine quantities:

- the distance in $r$ - $\phi$ between the two tracks at their point of tangency;

- the radius of the first measured hit of both tracks, as well as the radius of their common vertex;

- the invariant mass of the pair assuming both tracks to be electrons and the impact parameter of the parent photon with respect to the primary vertex of the event;

- the two track momenta;

- the output $\mathrm{NET}_{\mathrm{el}}$ of the partner track.

These quantities are fed into a separate neural network which achieves a tagging efficiency of $(90 \pm 2) \%$. The error is systematic and was derived from studies of photon conversions in radiative muon pair events. Most electrons from Dalitz decays, which represent a small contribution compared to photon conversions, are also rejected. Less than $4 \%$ of prompt electrons are wrongly tagged as conversions.

\subsection{Muon selection}

The muon identification is the same as in [22]. It relies mainly on the quality of the match between a track segment reconstructed in the muon chambers and the extrapolation of a track reconstructed in the central detector. A loose cut on $\mathrm{d} E / \mathrm{d} x$ is also used to reduce the kaon background. For this measurement, muons are selected in the full $\cos \theta$ range, and are required to have $p>4 \mathrm{GeV}$. 
The muon tagging efficiency and background level were tested using various samples of identified muons and hadrons: muon pairs from $Z^{0}$ decays and two-photon scattering, as well as pions from $\mathrm{K}^{0}$ and three-prong $\tau$ decays. In all cases, the simulation was found to give reliable predictions of the performances of the muon identification procedure. In the chosen momentum range, the tagging efficiency was estimated to be $(78 \pm 2) \%$, and the remaining hadronic background $(25 \pm 4) \%$, where the error is systematic.

\subsection{Summary}

Table 2 shows the composition of the electron and muon samples separately after all selections. Three main sources of prompt leptons are distinguished on the basis of the weakly decaying hadron: "right sign' $b$ decays, globally referred to as ' $b \rightarrow \ell^{-}$' (which include $b \rightarrow \ell^{-}, b \rightarrow \bar{c} \rightarrow \ell^{-}$and $b \rightarrow \tau \rightarrow \ell^{-}$decay chains $\left.^{2}\right)$; c decays, denoted ' $c \rightarrow \ell^{+}$', and cascade decays, referring to the process ' $b \rightarrow c \rightarrow \ell^{+}$'.

The 'non-prompt' background consists of leptons other than those produced in $b$ and $c$ hadron decays: electrons originating from photon conversion, Dalitz or light quark decays, muons produced in decays in flight of kaons and pions, etc.

\begin{tabular}{ccc} 
& electrons & muons \\
\hline $\mathrm{b} \rightarrow \ell^{-}$ & $43.3 \%$ & $37.0 \%$ \\
\hline $\mathrm{c} \rightarrow \ell^{+}$ & $21.1 \%$ & $19.2 \%$ \\
\hline $\mathrm{b} \rightarrow \mathrm{c} \rightarrow \ell^{+}$ & $14.8 \%$ & $10.0 \%$ \\
\hline non-prompt & $11.9 \%$ & $9.2 \%$ \\
\hline hadrons & $8.9 \%$ & $24.6 \%$ \\
\hline \hline total & 172289 & 189601 \\
\hline
\end{tabular}

Table 2: Estimated composition of the single electron and muon samples after the lepton selection.

\section{Variables for Flavour Separation}

Candidate leptons are classified into four distinct types, numbered as follows:
1. $\mathrm{b} \rightarrow \ell^{-}$,
2. $c \rightarrow \ell^{+}$,
3. $\mathrm{b} \rightarrow \mathrm{c} \rightarrow \ell^{+}$,
4. non-prompt leptons and misidentified hadrons.

The separation of these different contributions has been previously performed using the two variables that best describe the kinematical properties of the candidate lepton: its momentum $p$, and transverse momentum $p_{t}$ with respect to the nearest jet axis. In direct decays the lepton momentum reflects the hard fragmentation of the primary meson and is thus particularly efficient for separating contributions 1 and 2 from the others. The large mass of the b quark also induces high lepton energies in the rest frame of the decaying b-hadrons, which translates, unaffected by the boost along the quark (jet) direction, into hard $p_{t}$ spectra characteristic of direct and cascade b decays (classes 1 and 3 ), as can be seen from figure 4 .

\footnotetext{
${ }^{2}$ Charge conjugates are implied by default for all decay chains: for instance $\mathrm{b} \rightarrow \ell^{-}$refers also to $\overline{\mathrm{b}} \rightarrow \ell^{+}, \mathrm{b} \rightarrow \overline{\mathrm{c}} \rightarrow \ell^{-}$to $\overline{\mathrm{b}} \rightarrow \mathrm{c} \rightarrow \ell^{+}$, etc.
} 
Various combinations of these two basic variables have been used in the past, either to reduce the dimensionality of a fit [23] or to optimize the separation between two given classes [3]. As was recently demonstrated [24], the use of additional new variables, i.e. other than purely kinematical ones, can significantly improve the separation between the different lepton sources, provided these variables are properly combined.

For the measurement of the $\mathrm{b}$ and $\mathrm{c}$ quark asymmetries, it is especially important to separate direct $\mathrm{b}$ and $\mathrm{c}$ decays (types 1 and 2) from each other, and from all other contributions. Cascade decays dilute both the asymmetries and mixing, and are thus considered as being part of the background for flavour separation. In order to optimize the separation, as much of the available information as possible is used, including jet and vertex properties which have already proven efficient in heavyflavour tagging techniques. Here, many of these variables are combined with $p$ and $p_{t}$ with the help of neural networks, and the optimal combination giving the best separation is retained.

To isolate b decays from all other sources, a variable denoted $\mathrm{NET}_{\mathrm{b}}$ is constructed from a set of 5 discriminating variables:

- $p$, the track momentum;

- $p_{t}$, the transverse momentum with respect to the nearest jet axis (with the lepton excluded from the jet axis calculation);

- $\mathrm{E}_{\text {sub-jet }}$, the energy of the lepton sub-jet;

- $\mathrm{E}_{j e t}^{\text {vis }}$, the total visible energy of the jet;

- $\left(\sum p_{t}\right)_{j e t}$, the scalar sum of the transverse momentum of all tracks within the jet.

The concept of a lepton sub-jet as used here was first introduced in [24]. In each jet containing a candidate lepton, an iterative procedure is used to construct a sub-jet which, in simple terms, consists of tracks which are nearer the lepton direction than the jet axis. The presence of a sub-jet is more especially characteristic of cascade decays, and the sub-jet energy can be used as a measure of the lepton isolation which, unlike $p_{t}$, does not depend on the lepton momentum.

The total jet energy $\mathrm{E}_{j e t}^{\text {vis }}$ is useful since $\mathrm{b}$ jets where the $\mathrm{b}$ hadron has decayed semileptonically tend to have lower visible energy due to the emission of an energetic neutrino. The variable $\left(\sum p_{t}\right)_{j e t}$ characterizes both the width and multiplicity of the jet, which are known to differ significantly for $b$ quarks compared to lighter quarks [25].

To separate direct $\mathrm{c}$ decays from the cascade and non-prompt contributions, the optimal discriminating variable called $\mathrm{NET}_{\mathrm{c}}$ is constructed by combining 5 (only 3 for muons) additional measured quantities to the 5 previously listed:

- $\left(L / \sigma_{L}\right)_{1,2}$, the decay length significance of the jet containing the lepton (jet number 1 ) as well as that of the other most energetic jet in the event (jet number 2);

- $d / \sigma_{d}$ the impact parameter significance of the lepton with respect to the primary vertex;

- the outputs $\mathrm{NET}_{\mathrm{el}}$ and $\mathrm{NET}_{\mathrm{cv}}$ of the electron and photon conversion neural net taggers respectively (for electrons only).

Secondary vertices are reconstructed for each jet in the event using the same algorithm as in [7]. The decay length significance is defined as the distance $L$ of the secondary vertex from the primary vertex, divided by the estimated error $\sigma_{L}$ on $L$, which comes from the uncertainties in the primary and secondary vertex positions. For each track, the impact parameter significance is the ratio of the distance $d$ between the primary vertex and the track at the point of closest approach, to the error $\sigma_{d}$ on this distance. Both the decay length and the impact parameter are defined in the $r$ - $\phi$ plane only. $\left(L / \sigma_{L}\right)_{1,2}$ are useful to separate $\mathrm{c}$ meson decays from both cascade and light meson decays which have 
very different lifetimes, while the impact parameter allows the distinction between secondary tracks and tracks from fragmentation.

Although the variables $\mathrm{NET}_{\mathrm{el}}$ and $\mathrm{NET}_{\mathrm{cv}}$ have already been used to remove the bulk of the hadronic and photon conversion components, they remain useful for further separation of prompt electrons from these two types of background.

Figure 5 shows the expected distribution for the four types of candidate electrons in the plane of the two separating variables called respectively $\mathrm{NET}_{\mathrm{b}}$ and $\mathrm{NET}_{\mathrm{c}}$. The direct $\mathrm{b}$ and $\mathrm{c}$ decay contributions are clearly separated from each other and from other contributions. The comparison with figure 4 demonstrates the gain resulting from the use of additional variables. Table 3 shows the typical composition of two samples enriched in leptons from either direct $\mathrm{b}$ or $\mathrm{c}$ decays. These samples contain respectively $52 \%$ and $21 \%$ of all $\mathrm{b} \rightarrow \ell^{-}$and $\mathrm{c} \rightarrow \ell^{+}$passing the lepton selection. The $\mathrm{NET}_{\mathrm{b}}$ and $\mathrm{NET}_{\mathrm{c}}$ distributions obtained from data and from Monte Carlo are represented in figure 6.

\begin{tabular}{lcc} 
& $\mathrm{b} \rightarrow \ell^{-}$enriched & $\mathrm{c} \rightarrow \ell^{+}$enriched \\
\hline & $\mathrm{NET}_{\mathrm{b}}>0.8$ & $\mathrm{NET}_{\mathrm{b}}<0.3$ and $\mathrm{NET}_{\mathrm{c}}>0.7$ \\
\hline $\mathrm{b} \rightarrow \ell^{-}$ & $85.3 \%$ & $10.1 \%$ \\
\hline $\mathrm{c} \rightarrow \ell^{+}$ & $3.5 \%$ & $56.7 \%$ \\
\hline $\mathrm{b} \rightarrow \mathrm{c} \rightarrow \ell^{+}$ & $4.3 \%$ & $9.3 \%$ \\
\hline others & $6.9 \%$ & $23.9 \%$ \\
\hline
\end{tabular}

Table 3: Composition of $\mathrm{b} \rightarrow \ell^{-}$and $\mathrm{c} \rightarrow \ell^{+}$enriched samples.

\section{The Fitting Method}

In each event, the thrust axis is used to estimate the quark direction, and the quark charge is inferred from the lepton charge $Q_{\ell}$. The observable $y$ is defined as $-Q_{\ell} \cdot \cos \theta_{\text {thrust }}$, where $\cos \theta_{\text {thrust }}$ denotes the cosine of the thrust axis oriented positively along the lepton direction. An event with $y>0$ $(y<0)$ is said to be forward (backward). The asymmetries $A_{\mathrm{FB}}^{\mathrm{b}}$ and $A_{\mathrm{FB}}^{\mathrm{c}}$ are extracted from single lepton events using a binned maximum likelihood fit, by comparing the differential forward-backward asymmetry as a function of $|y|$ measured in the data to the expected asymmetry. Dilepton events are explicitly excluded from the single lepton sample. The average mixing parameter $\bar{\chi}$ is obtained simultaneously by counting the number of like-sign events in the dilepton sample.

The total likelihood to be maximized is the product:

$$
\mathcal{L}=\mathcal{L}_{\text {single }} \times \mathcal{L}_{\text {double }}
$$

where the likelihood for single-lepton events has the form:

$$
\mathcal{L}_{\text {single }}=\prod_{\mathrm{NET}_{\mathrm{b}}, \mathrm{NET}_{c}} \prod_{|y|} \frac{\left(n_{F}+n_{B}\right) !}{n_{F} ! n_{B} !} \times\left(\frac{1+A_{\mathrm{FB}}}{2}\right)^{n_{F}} \times\left(\frac{1-A_{\mathrm{FB}}}{2}\right)^{n_{B}} .
$$

The quantities $n_{F}$ and $n_{B}$ are respectively the number of forward and backward events in a bin ${ }^{3}$ of $\mathrm{NET}_{\mathrm{b}}, \mathrm{NET}_{\mathrm{c}}$ and $|y|$. The number of forward events $n_{F}$ follows a binomial distribution of probability $\left(1+A_{\mathrm{FB}}\right) / 2$ where $A_{\mathrm{FB}}$ is the expected forward-backward asymmetry in the bin considered:

$$
A_{\mathrm{FB}}\left(\mathrm{NET}_{\mathrm{b}}, \mathrm{NET}_{\mathrm{c}},|y|\right)=\sum_{i=1}^{4} f_{i}\left(\mathrm{NET}_{\mathrm{b}}, \mathrm{NET}_{\mathrm{c}},|y|\right) A_{\mathrm{FB}}^{i} \frac{8}{3} \frac{|y|}{1+|y|^{2}} .
$$

\footnotetext{
${ }^{3} 10 \times 10$ bins in the $\left(\mathrm{NET}_{\mathrm{b}}, \mathrm{NET}_{c}\right)$ plane and 10 bins along $|y|$ are used
} 
In this expression, $f_{i}$ denotes the predicted fraction of lepton of type $i$ and $A_{\mathrm{FB}}^{i}$ represents the corresponding theoretical asymmetry:

$$
\left\{\begin{array}{cccl}
A_{\mathrm{FB}}^{1}= & (1-2 \bar{\chi}) & A_{\mathrm{FB}}^{\mathrm{b}} & \text { for } \mathrm{b} \rightarrow \ell^{-}, \\
A_{\mathrm{FB}}^{2}= & - & A_{\mathrm{FB}}^{\mathrm{C}} & \text { for } \mathrm{c} \rightarrow \ell^{+}, \\
A_{\mathrm{FB}}^{3}= & -(1-2 \bar{\chi}) & A_{\mathrm{FB}}^{\mathrm{b}} & \text { for } \mathrm{b} \rightarrow \mathrm{c} \rightarrow \ell^{+}, \\
A_{\mathrm{FB}}^{4}= & & 0 & \text { for background } .
\end{array}\right.
$$

The fractions $f_{i}$ are derived from the knowledge of the misidentification probability of the lepton selection algorithms, the partial widths $\Gamma_{\mathrm{b} \overline{\mathrm{b}}}$ and $\Gamma_{\mathrm{c} \overline{\mathrm{c}}}$ and the semileptonic decay branching ratios, and from the $\left(\mathrm{NET}_{\mathrm{b}}, \mathrm{NET}_{\mathrm{c}}\right)$ distribution taken from the simulation. $A_{\mathrm{FB}}^{\mathrm{b}}, A_{\mathrm{FB}}^{\mathrm{c}}$ and $\bar{\chi}$ are the only free parameters. $\bar{\chi}$ is constrained by the likelihood computed from dilepton events:

$$
\mathcal{L}_{\text {double }}=\frac{\left(n_{\ell^{ \pm} \ell^{ \pm}}+n_{\ell_{\ell}^{ \pm}}\right) !}{n_{\ell^{ \pm} \ell^{ \pm}} ! n_{\ell^{ \pm} \ell \mp} !} R^{n_{\ell^{ \pm} \ell^{ \pm}}}(1-R)^{n_{\ell \pm \ell \mp}},
$$

where $n_{\ell^{ \pm} \ell^{ \pm}}$and $n_{\ell^{ \pm} \ell^{\mp}}$ are respectively the number of like-sign and opposite-sign events. $n_{\ell^{ \pm} \ell^{ \pm}}$obeys a binomial law of parameter $R$, which is a function of $\bar{\chi}$ :

$$
\begin{array}{rlcc}
R=2 f_{11} \bar{\chi}(1-\bar{\chi}) & +f_{13}\left(\bar{\chi}^{2}+(1-\bar{\chi})^{2}\right) & + & f_{14} / 2 \\
& +2 f_{33} \bar{\chi}(1-\bar{\chi}) & + & f_{34} / 2 \\
& & + & \left(f_{24}+f_{44}\right) / 2 .
\end{array}
$$

The notation $f_{i j}$ is used for the fraction of dilepton events containing one lepton of type $i$ and one lepton of type $j(i=1,4$ and $j=1,4)$. Events of type $f_{13}$ are the dominant background to events with both leptons from direct b decays $\left(f_{11}\right)$ and reduce the sensitivity of $R$ to the mixing parameter. To minimize this effect, as well as to reduce systematic uncertainties, cascade decays are rejected from the dilepton sample by requiring that both leptons satisfy: $\mathrm{NET}_{\mathrm{b}}>0.8$. This cut has been chosen so as to minimize the total error (statistical plus systematic) on the mixing measurement. The composition of the dilepton sample after this cut is indicated in table 4 separately for ee, e $\mu$ and $\mu \mu$ events.

\begin{tabular}{cccc} 
& $\mathrm{ee}$ & $\mu \mu$ & $\mathrm{e} \mu$ \\
\hline$\left(\mathrm{b} \rightarrow \ell^{-}, \mathrm{b} \rightarrow \ell^{-}\right)$ & $89.6 \%$ & $85.5 \%$ & $89.6 \%$ \\
\hline$\left(\mathrm{b} \rightarrow \ell^{-}, \mathrm{b} \rightarrow \mathrm{c} \rightarrow \ell^{+}\right)$ & $7.7 \%$ & $7.5 \%$ & $5.5 \%$ \\
\hline$\left(\mathrm{b} \rightarrow \mathrm{c} \rightarrow \ell^{+}, \mathrm{b} \rightarrow \mathrm{c} \rightarrow \ell^{+}\right)$ & $0.2 \%$ & $0.1 \%$ & $0.2 \%$ \\
\hline others & $2.5 \%$ & $6.9 \%$ & $4.7 \%$ \\
\hline \hline Total events & 626 & 920 & 1449 \\
\hline
\end{tabular}

Table 4: Estimated composition of the 3 dilepton samples after requiring $\mathrm{NET}_{\mathrm{b}}>0.8$ for both leptons.

\section{$7 \quad$ Fit Results}

The fit results based on the full lepton sample are given in table 5. The fit has also been performed separately for electrons and for muons. All results are consistent within their statistical errors. Note that the mixing is always measured from the entire data sample, including off-peak dilepton events. The correlation matrices are given in table 6 .

The fit method is illustrated in figure 7 for the $b \rightarrow \ell^{-}$and $c \rightarrow \ell^{+}$enriched samples defined in table 3 . The observed asymmetry $\left(n_{F}-n_{B}\right) /\left(n_{F}+n_{B}\right)$ is compared to the fitted function $8 A_{\mathrm{FB}}(|y|) / 3\left(1+y^{2}\right)$ in bins of $|y|$. When fitting the $b \rightarrow \ell^{-}$enriched sample, the c asymmetry is fixed to its Standard Model 
value. Similarly, for the $c \rightarrow \ell^{+}$enriched sample, the $b$ asymmetry is kept constant. The results are in good agreement with those obtained from the full range fit, as can be seen from table 7 .

As a simple cross-check, the measurement of $A_{\mathrm{FB}}^{\mathrm{b}}$ and $\bar{\chi}$ has been repeated using only high $p_{t}$ leptons, $\left(p_{t}>1.2 \mathrm{GeV} / c\right.$, where $p_{t}$ is computed with respect to the nearest jet including the lepton), with the $c$ asymmetry being fixed as previously to its Standard Model value. In this case, the fractions $f_{i}$ are simple constants, and the fit does not rely on the modelling of the $\mathrm{NET}_{\mathrm{b}}$ and $\mathrm{NET}_{\mathrm{c}}$ variables. As can be seen from table 7 , the fitted values are also in good agreement with our previous results, though with larger statistical errors.

\begin{tabular}{|cc|c|c||c|}
\hline \multicolumn{2}{|c|}{ Full range fit } & Electrons & Muons & Combined \\
\hline peak & $A_{\mathrm{FB}}^{\mathrm{b}}$ & $8.83 \pm 0.73$ & $9.48 \pm 0.73$ & $9.06 \pm 0.51$ \\
& $A_{\mathrm{FB}}^{\mathrm{c}}$ & $5.80 \pm 0.88$ & $5.65 \pm 0.96$ & $6.00 \pm 0.67$ \\
\hline peak-2 & $A_{\mathrm{FB}}^{\mathrm{b}}$ & $1.9 \pm 3.4$ & $8.7 \pm 3.5$ & $5.5 \pm 2.4$ \\
& $A_{\mathrm{FB}}^{\mathrm{c}}$ & $-6.0 \pm 4.5$ & $-9.0 \pm 4.8$ & $-7.5 \pm 3.4$ \\
\hline peak+2 & $A_{\mathrm{FB}}^{\mathrm{b}}$ & $8.6 \pm 3.0$ & $14.6 \pm 2.8$ & $11.7 \pm 2.0$ \\
& $A_{\mathrm{FB}}^{\mathrm{c}}$ & $13.9 \pm 3.7$ & $14.0 \pm 4.0$ & $14.1 \pm 2.8$ \\
\hline all & $\bar{\chi}$ & $0.1213 \pm 0.0106$ & $0.1082 \pm 0.0101$ & $0.1107 \pm 0.0062$ \\
\hline
\end{tabular}

Table 5: Comparison of the electron, muon and combined results for the full range fit. The asymmetries (in \%) are measured at the three centre-of-mass energy points denoted peak, peak-2 and peak +2 (table 1 ), whereas the mixing $\bar{\chi}$ is obtained using the full data sample. Errors are statistical only.

\begin{tabular}{|c|c|c|c|}
\hline peak & $A_{\mathrm{FB}}^{\mathrm{b}}$ & $A_{\mathrm{FB}}^{\mathrm{c}}$ & $\bar{\chi}$ \\
\hline$A_{\mathrm{FB}}^{\mathrm{b}}$ & 1.00 & 0.18 & 0.29 \\
$A_{\mathrm{FB}}^{\mathrm{c}}$ & & 1.00 & 0.00 \\
$\bar{\chi}$ & & & 1.00 \\
\hline
\end{tabular}

\begin{tabular}{|c|c|c|c|}
\hline peak -2 & $A_{\mathrm{FB}}^{\mathrm{b}}$ & $A_{\mathrm{FB}}^{\mathrm{c}}$ & $\bar{\chi}$ \\
\hline$A_{\mathrm{FB}}^{\mathrm{b}}$ & 1.00 & 0.20 & 0.04 \\
$A_{\mathrm{FB}}^{\mathrm{c}}$ & & 1.00 & 0.00 \\
$\bar{\chi}$ & & & 1.00 \\
\hline
\end{tabular}

\begin{tabular}{|c|c|c|c|}
\hline peak +2 & $A_{\mathrm{FB}}^{\mathrm{b}}$ & $A_{\mathrm{FB}}^{\mathrm{c}}$ & $\bar{\chi}$ \\
\hline$A_{\mathrm{FB}}^{\mathrm{b}}$ & 1.00 & 0.19 & 0.09 \\
$A_{\mathrm{FB}}^{\mathrm{c}}$ & & 1.00 & 0.00 \\
$\bar{\chi}$ & & & 1.00 \\
\hline
\end{tabular}

Table 6: Statistical correlation matrices at each centre-of-mass energy.

\begin{tabular}{|cc|c|c|c|c|}
\hline & & Full range fit & $\mathrm{b} \rightarrow \ell^{-}$enriched & $\mathrm{c} \rightarrow \ell^{+}$enriched & High $p_{t}$ fit \\
\hline peak & $A_{\mathrm{FB}}^{\mathrm{b}}$ & $9.06 \pm 0.51$ & $8.98 \pm 0.57$ & 9.1 (fixed) & $9.28 \pm 0.67$ \\
& $A_{\mathrm{FB}}^{\mathrm{c}}$ & $6.00 \pm 0.67$ & 6.0 (fixed) & $7.78 \pm 1.10$ & 6.0 (fixed) \\
\hline peak-2 & $A_{\mathrm{FB}}^{\mathrm{b}}$ & $5.5 \pm 2.4$ & $6.4 \pm 2.7$ & 5.4 (fixed) & $1.6 \pm 3.3$ \\
& $A_{\mathrm{FB}}^{\mathrm{c}}$ & $-7.5 \pm 3.4$ & -3.3 (fixed) & $-8.9 \pm 5.6$ & -3.3 (fixed) \\
\hline peak +2 & $A_{\mathrm{FB}}^{\mathrm{b}}$ & $11.7 \pm 2.0$ & $11.9 \pm 2.3$ & 11.4 (fixed) & $11.4 \pm 2.7$ \\
& $A_{\mathrm{FB}}^{\mathrm{c}}$ & $14.1 \pm 2.8$ & 11.9 (fixed) & $3.4 \pm 4.7$ & 11.9 (fixed) \\
\hline all & $\bar{\chi}$ & $0.1107 \pm 0.0062$ & $0.1110 \pm 0.0062$ & $0.1104 \pm 0.0062$ & $0.1049 \pm 0.0090$ \\
\hline
\end{tabular}

Table 7: Comparison of the results obtained from the full range fit, the $\mathrm{b} \rightarrow \ell^{-}$and $\mathrm{c} \rightarrow \ell^{+}$enriched samples and the high $p_{t}$ fit. Whenever $A_{\mathrm{FB}}^{\mathrm{b}}$ or $A_{\mathrm{FB}}^{\mathrm{c}}$ are kept fixed, the indicated value corresponds to the Standard Model prediction as calculated by ZFITTER [26], with $m_{\mathrm{top}}=180 \mathrm{GeV} / c^{2}$ and $m_{\mathrm{H}}=300 \mathrm{GeV} / c^{2}$. Errors are statistical only. 


\section{Systematic Uncertainties}

Three types of systematic uncertainties have been considered: those coming from the models and theoretical predictions used in the measurement, those induced by detector effects, and finally various possible systematic effects related to the fitting method.

\subsection{Phenomenological models and theoretical predictions}

The first type of systematic effect comes from the use of phenomenological models to describe the fragmentation of heavy quarks as well as the semileptonic decays of heavy mesons. These also include uncertainties in the values of $Z^{0}$ partial decay widths and semileptonic decay branching ratios taken either from theoretical predictions or independent measurements [27].

\section{Fragmentation}

In the simulation, the fragmentation of heavy quarks is described by the fragmentation function of Peterson et al. [15]. The parameters controlling the shape of the fragmentation function were set respectively to $\epsilon_{\mathrm{b}}=0.0055$ and $\epsilon_{\mathrm{c}}=0.070$ for $\mathrm{b} \overline{\mathrm{b}}$ and $c \overline{\mathrm{c}}$ events, corresponding to LEP average values of the mean scaled energy $\left\langle x_{E}\right\rangle_{\mathrm{b}}=0.70$ and $\left\langle x_{E}\right\rangle_{\mathrm{c}}=0.49[3,6,23,28]$.

Systematic uncertainties are obtained by varying the fragmentation parameters between 0.0025 and 0.0095 for $b$ quarks and between 0.050 and 0.100 for $c$ quarks. The chosen range of variation for $\epsilon_{\mathrm{b}}$ and $\epsilon_{\mathrm{c}}$ corresponds to an uncertainty of \pm 0.02 for both $\left\langle x_{E}\right\rangle_{\mathrm{b}}$ and $\left\langle x_{E}\right\rangle_{\mathrm{c}}$, which is significantly larger than the experimental error on present measurements, and also accounts for uncertainties in the choice of the model itself.

\section{Semileptonic decay models}

The semileptonic decays of heavy hadrons are described by the free-quark model of Altarelli et al. (ACCMM) [16] which has two free parameters: the Fermi momentum $p_{\mathrm{f}}$ and the mass of the quark produced in the decay of the heavy hadron: $m_{\mathrm{c}}$ or $m_{\mathrm{s}}$. For b decays, values of $p_{\mathrm{f}}=298 \mathrm{MeV} / c$ and $m_{c}=1673 \mathrm{MeV} / c^{2}$ have been obtained from a fit to CLEO data [17], while for $c$ decays, the combined measurements of DELCO [18] and MARK III [19] have been used to derive values of $p_{\mathrm{f}}=0.467 \mathrm{GeV} / \mathrm{c}$ and $m_{\mathrm{s}}=0.001 \mathrm{GeV} / c^{2}$.

Systematic uncertainties arising from the choice of the semileptonic decay model are treated differently for $b$ and $c$ hadron decays by varying either the model or its parameters:

- For $\mathrm{b} \rightarrow \ell^{-}$decays, the form-factor model of Isgur et al. [29] provides an alternative parametrization of the lepton momentum in the rest frame of the decaying $\mathrm{B}$ hadron. This model (ISGW) has no free parameter but was found to describe CLEO data better when the fraction of $b$ decays to $\mathrm{D}^{* *}$ was set to $32 \%$ instead of $11 \%$ as calculated in [29]. This modified model, denoted ISGW $^{* *}$, is thus also used as a possible alternative.

- Uncertainties affecting the modelling of $c \rightarrow \ell^{+}$decay can be estimated by using various versions of the ACCMM model based on different sets of values for the fitted parameters $m_{\mathrm{s}}$ and $p_{\mathrm{f}}$. The results using the central values $m_{\mathrm{s}}=0.001 \mathrm{GeV} / c^{2}$ and $p_{\mathrm{f}}=0.467 \mathrm{GeV} / c$ are compared to the results obtained with:

$$
\begin{aligned}
& -m_{\mathrm{s}}=0.001 \mathrm{GeV} / c^{2} \text { and } p_{\mathrm{f}}=0.353 \mathrm{GeV} / c(\mathrm{ACCMM} 1), \\
& -m_{\mathrm{s}}=0.153 \mathrm{GeV} / c^{2} \text { and } p_{\mathrm{f}}=0.467 \mathrm{GeV} / c(\text { ACCMM2). }
\end{aligned}
$$

where the chosen range of variation reflects the uncertainty in the fitted values of $m_{\mathrm{s}}$ and $p_{\mathrm{f}}$.

These models are varied independently for direct $b$ and $c$ decays. For cascade decays $b \rightarrow c \rightarrow \ell^{+}$, the $\mathrm{D}$ momentum spectrum measured by CLEO [30] is combined with the $\mathrm{c} \rightarrow \ell^{+}$model to generate the 
lepton momentum distribution. Uncertainties in the CLEO $\mathrm{b} \rightarrow \mathrm{D}$ spectrum are negligible compared to the effect of varying $m_{\mathrm{s}}$ and $p_{\mathrm{f}}$.

\section{Branching ratios}

The values of $\mathrm{BR}\left(\mathrm{b} \rightarrow \ell^{-}\right)$and $\mathrm{BR}\left(\mathrm{b} \rightarrow \mathrm{c} \rightarrow \ell^{+}\right)$given in table 8 are derived from independent measurements performed by ALEPH, OPAL and DELPHI [3,23,31], which are combined using the procedure described in [1]. Part of this procedure is to propagate through the measurements a consistent choice of input parameters for systematic errors. In the measurements of $\mathrm{BR}\left(\mathrm{b} \rightarrow \ell^{-}\right)$and $\mathrm{BR}\left(\mathrm{b} \rightarrow \mathrm{c} \rightarrow \ell^{+}\right)$, an important part of the error is due to the choice of semileptonic decay model. This error is indicated in parentheses in table 8 . It is taken into account in a self consistent manner by using the branching ratios which correspond to each choice of semileptonic decay model as the model is varied. The first part of the error (i.e. not due to the models) is then used to estimate the additional systematic error due to the uncertainty in $\mathrm{BR}\left(\mathrm{b} \rightarrow \ell^{-}\right)$and $\mathrm{BR}\left(\mathrm{b} \rightarrow \mathrm{c} \rightarrow \ell^{+}\right)$, excluding the model uncertainty.

$\mathrm{BR}\left(\mathrm{c} \rightarrow \ell^{+}\right)$is taken from lower energy experiments. Measurements from ARGUS, PEP and PETRA [32] are combined to give $\mathrm{BR}\left(\mathrm{c} \rightarrow \ell^{+}\right)=(9.8 \pm 0.5) \%$. The value of $\mathrm{BR}\left(\mathrm{b} \rightarrow \tau \rightarrow \ell^{-}\right)$is derived from the $b \rightarrow \tau$ decay rate measured by ALEPH [33] and from world average values of the tau leptonic branching ratios [34]. The JETSET 7.3 prediction [12] for $\mathrm{BR}\left(\mathrm{b} \rightarrow \overline{\mathrm{c}} \rightarrow \ell^{-}\right)$of $1.3 \%$ is used for the central value, and the error of $0.5 \%$ allows for $(15 \pm 5) \%$ of b-quark decays to produce a $\bar{c}$ anti-quark, and a $15 \%$ uncertainty in the semileptonic branching ratio of the resulting charm hadrons.

\begin{tabular}{|l|c|}
\hline \multicolumn{2}{|c|}{ Branching ratios (in \%) } \\
\hline $\mathrm{BR}\left(\mathrm{b} \rightarrow \ell^{-}\right)$ & $10.90 \pm 0.32(\mp 0.21)$ \\
$\mathrm{BR}\left(\mathrm{b} \rightarrow \mathrm{c} \rightarrow \ell^{+}\right)$ & $8.30 \pm 0.47( \pm 0.19)$ \\
$\mathrm{BR}\left(\mathrm{b} \rightarrow \overline{\mathrm{c}} \rightarrow \ell^{-}\right)$ & $1.30 \pm 0.50$ \\
$\mathrm{BR}\left(\mathrm{b} \rightarrow \tau \rightarrow \ell^{-}\right)$ & $0.70 \pm 0.20$ \\
$\mathrm{BR}\left(\mathrm{c} \rightarrow \ell^{+}\right)$ & $9.80 \pm 0.50$ \\
\hline
\end{tabular}

Table 8: Values of branching ratios used for the semileptonic decays of heavy quarks. For BR(b $\left.\rightarrow \ell^{-}\right)$ and $\operatorname{BR}\left(b \rightarrow c \rightarrow \ell^{+}\right)$, the first quoted error is the total experimental error, without the error due to the semileptonic decay model which is given in parentheses (the $+1 \sigma$ numbers correspond to a change of model from ACCMM to ISGW).

\section{Partial widths}

The ratios $R_{\mathrm{b}}=\Gamma_{\mathrm{b} \overline{\mathrm{b}}} / \Gamma_{\text {hadron }}$ and $R_{\mathrm{c}}=\Gamma_{\mathrm{c} \overline{\mathrm{c}}} / \Gamma_{\text {hadron }}$ are fixed at their Standard Model values $\left(R_{\mathrm{b}}^{S M}=\right.$ 0.2155 and $R_{c}^{S M}=0.172$ ) as predicted by ZFITTER [26]. The dependence of the fitted asymmetries on $R_{\mathrm{b}}$ and $R_{\mathrm{c}}$ can be parametrized in the form:

$$
\left\{\begin{array}{l}
\Delta A_{\mathrm{FB}}^{\mathrm{b}}=a_{\mathrm{b}}\left(R_{\mathrm{q}}\right) \frac{\Delta R_{\mathrm{q}}}{R_{\mathrm{q}}^{S M}} \\
\Delta A_{\mathrm{FB}}^{\mathrm{c}}=a_{\mathrm{c}}\left(R_{\mathrm{q}}\right) \frac{\Delta R_{\mathrm{q}}}{R_{\mathrm{q}}^{S M}}
\end{array}\right.
$$

where $\Delta R_{\mathrm{q}}=R_{\mathrm{q}}-R_{\mathrm{q}}^{S M}$ for $\mathrm{q}=\mathrm{b}, \mathrm{c}$. The values of the coefficients $a_{\mathrm{b}}\left(R_{\mathrm{q}}\right)$ and $a_{\mathrm{c}}\left(R_{\mathrm{q}}\right)$ are given in table 9 for the on-peak and off-peak results. The average mixing parameter $\bar{\chi}$ is essentially insensitive to small variations of $R_{\mathrm{b}}$ or $R_{\mathrm{c}}$. 


\begin{tabular}{|c|c|c|c|}
\hline & on-peak & peak-2 & peak +2 \\
\hline$a_{\mathrm{b}}\left(R_{\mathrm{b}}\right)$ & -0.009 & -0.004 & -0.015 \\
$a_{\mathrm{c}}\left(R_{\mathrm{b}}\right)$ & +0.031 & -0.023 & +0.061 \\
\hline$a_{\mathrm{b}}\left(R_{\mathrm{c}}\right)$ & +0.009 & +0.003 & +0.014 \\
$a_{\mathrm{c}}\left(R_{\mathrm{c}}\right)$ & -0.030 & +0.024 & -0.061 \\
\hline
\end{tabular}

Table 9: Values of the coefficients $a_{\mathrm{b}}\left(R_{\mathrm{q}}\right)$ and $a_{\mathrm{c}}\left(R_{\mathrm{q}}\right)$ showing the dependence of the fitted asymmetries on $R_{\mathrm{b}}$ and $R_{\mathrm{c}}$.

\subsection{Detector effects}

A second type of systematic effect comes from uncertainties in the lepton selection as well as in the distributions of the variables used in the fit which are mainly caused by uncertainties in the modelling of the detector's response.

\section{Lepton backgrounds}

For electrons, the hadronic background is estimated from the misidentification probability predicted by the simulation which has been shown to reproduce the data over the full range of momentum and polar angle. The resulting Monte Carlo prediction is reliable to $\pm 15 \%$.

The estimation of the number of untagged conversions in the data is based on the observed number of tagged conversions after the electron selection and on the knowledge of the tagging efficiency of the conversion finder. This efficiency $(\approx 90 \%)$ measured from the simulation, was found to be correct to $2 \%$ using photon conversions in radiative muon pairs and by studying the effect of variations of the tracking resolution in the simulation. This uncertainty translates into a $\pm 20 \%$ systematic error on the remaining fraction of untagged conversions.

For the muon selection, the hadronic background level is understood to within $\pm 15 \%$. An error of $\pm 10 \%$ is also assigned to the fraction of non-prompt muons (muons from pion and kaon decays in flight).

\section{Source dependence}

The electron and muon selections are both more efficient for isolated leptons, resulting in a greater efficiency for leptons produced in direct b decays compared to the other sources. This source dependence is particularly important for electrons, since most input variables used in $\mathrm{NET}_{\mathrm{el}}$ are very sensitive to the presence of nearby tracks.

Using electrons from photon conversions, this source dependence has been checked to be correctly simulated by comparing the efficiency of the electron selection in the data and in the simulation, in bins of the variables known to be the most sensitive to isolation. No deviation larger than the global $\pm 2.5 \%$ uncertainty on the prompt electron efficiency was observed. Checks were also performed using isolated electrons produced in two photon events $\gamma \gamma \rightarrow \mathrm{e}^{+} \mathrm{e}^{-}$, and confirmed that the efficiency obtained from the simulation is accurate within this uncertainty. The systematic error associated to the modelling of source dependence is thus estimated by varying individually the efficiency for each source of prompt electrons by $\pm 2.5 \%$.

\section{Tracking resolution}

The tracking resolution is known to be too optimistic in the Monte Carlo samples used in this analysis. A smearing algorithm has thus been applied before fitting, affecting especially the decay length and impact parameter distributions. The total effect of this smearing is used to estimate the systematic uncertainty due to the tracking resolution in the simulation. 


\section{Input modelling}

Some input variables used in the two neural networks $\mathrm{NET}_{\mathrm{b}}$ and $\mathrm{NET}_{\mathrm{c}}\left(\right.$ like $\mathrm{E}_{j e t}^{\text {vis }}, L / \sigma \ldots$ ) are sensitive to effects which have not been accounted for yet. These effects are globally referred to as "detector effects", though they can be due either to imperfections in the detector simulation or to physics (jet multiplicity, D mesons lifetimes...).

Some simple corrections (scaling factors) are derived for each of these variables by comparing the corresponding distribution for the whole lepton sample in the data and in the simulation. For instance the sub-jet energy is scaled up by a factor 1.01 in the simulation, and the variable $\left(\sum p_{t}\right)_{j e t}$ by a factor 1.02. The same scaling factors were always found to be valid for both electrons and muons. When for a given input this correction allows to improve the agreement of the $\mathrm{NET}_{\mathrm{b}}$ or $\mathrm{NET}_{\mathrm{c}}$ distributions between data and Monte Carlo, it is applied before fitting. In any case, the whole effect of the correction is used to estimate the systematic uncertainty due to the modelling of this variable.

\subsection{Other systematic effects}

\section{Composition dependence on $\cos \theta_{\text {thrust }}$}

In equation (3), the simulation is needed to predict the dependence of the relative fractions $f_{i}$ on $|y|=\left|\cos \theta_{\text {thrust }}\right|$. This dependence comes primarily from the fact that the performances of both the electron and muon selections vary with $\theta$. For instance, the electron sample has significantly higher hadronic background in a small region of $|\cos \theta|$ where the barrel and endcap overlap $(0.7<|\cos \theta|<$ 0.8). The number of conversions also increases with $|\cos \theta|$ due to the increasing amount of material. This $\cos \theta$ dependence induces a slight variation of the composition with $y$. Since it is difficult to check how well this variation is reproduced in the simulation, a $100 \%$ uncertainty is assigned to the Monte Carlo prediction, by comparing the results obtained with or without $\theta$ dependence.

The degradation of the tracking resolution with increasing $|y|$ values also affects the shape of the $\left(\mathrm{NET}_{\mathrm{b}}, \mathrm{NET}_{\mathrm{c}}\right)$ distribution, especially through the use of variables such as $\left(L / \sigma_{L}\right)_{1,2}$ and $d / \sigma_{d}$. Two different parametrizations of the $\left(\mathrm{NET}_{\mathrm{b}}, \mathrm{NET}_{\mathrm{c}}\right)$ distribution obtained respectively for $|y|<0.7$ and $|y|>0.7$ are used. The validity of these parametrizations is tested by varying the binning in $|y|$.

\section{Monte Carlo statistics}

Though the Monte Carlo and data samples used in this analysis have about the same number of events, statistical fluctuations in the Monte Carlo are reduced compared to data due to the additional binning of the data in $|y|$. To estimate the effect of these fluctuations the fit has been performed using 3 sub-samples of the entire Monte Carlo sample. The maximum difference between the results obtained with these sub-samples and with the whole sample is taken as the systematic uncertainty due to limited Monte Carlo statistics.

\section{Sensitivity to time-dependent mixing}

The presence of lifetime information in $\mathrm{NET}_{c}$ (decay length and impact parameter significance) might make the measurement of $A_{\mathrm{FB}}^{\mathrm{c}}$ and $A_{\mathrm{FB}}^{\mathrm{b}}$ sensitive to time-dependent mixing. Since this information is very diluted, the effect is expected to be small and is not corrected for. Still, it can be seen in the simulation that the fraction of mixed leptons (leptons coming from mixed B mesons) increases slightly at lower $\mathrm{NET}_{\mathrm{c}}$ (which corresponds in this case to longer decay lengths).

The simulation is used to estimate the effect of this variation. Monte Carlo tracks are randomly re-mixed in bins of $\mathrm{NET}_{c}$ so as to make the mixing constant as a function of $\mathrm{NET}_{c}$, without changing its average value. The fit is then performed on the re-mixed Monte Carlo sample and the values obtained for $A_{\mathrm{FB}}^{\mathrm{b}}$ and $A_{\mathrm{FB}}^{\mathrm{c}}$ are compared with those fitted from the original Monte Carlo sample. In order not to be sensitive to statistical fluctuations introduced by the random re-mixing itself, this procedure is repeated one hundred times, and the averages of the one hundred measurements of $A_{\mathrm{FB}}^{\mathrm{b}}$ and $A_{\mathrm{FB}}^{\mathrm{c}}$ are compared with the original values. The difference, found to be very small, is used as a 
systematic uncertainty.

\section{Background asymmetry}

The hadronic and non-prompt background have been assumed to have no asymmetry in the fit (equation (4)). This assumption has been checked and confirmed in different momentum ranges using the simulation. The background asymmetry has also been estimated directly from the data using only tracks which did not pass the lepton selection, or using all tracks in the momentum range $p>2 \mathrm{GeV} / c$ or $p>4 \mathrm{GeV} / c$ weighted by the fake probability predicted by the simulation in a given $\left(p, p_{t}\right)$ bin. All these tests indicated that the upper limit for any possible residual asymmetry of the background is $0.5 \%$. A $\pm 0.5 \%$ error was thus assigned to the background asymmetry.

A detailed list of systematic effects is given in tables 10 and 11.

\begin{tabular}{lccc}
\hline & $A_{\mathrm{FB}}^{\mathrm{b}}(\%)$ & $A_{\mathrm{FB}}^{\mathrm{c}}(\%)$ & $\bar{\chi}$ \\
\hline Fitted value & 9.06 & 6.00 & 0.1107 \\
\hline Statistical error & \pm 0.51 & \pm 0.67 & \pm 0.0062 \\
\hline Systematic error & \pm 0.23 & \pm 0.52 & \pm 0.0055 \\
\hline
\end{tabular}

Sources of systematic errors

\begin{tabular}{lccc}
\hline $\mathrm{b} \rightarrow \ell^{-}(\mathrm{ACCMM}$ to ISGWW \\
\hline $\left.\mathrm{c} \rightarrow \ell^{+}\right)$ & +0.06 & +0.24 & +0.0000 \\
\hline$\left\langle x_{E}\right\rangle_{\mathrm{b}}-0.02$ & +0.07 & -0.09 & -0.0022 \\
\hline$\left\langle x_{E}\right\rangle_{\mathrm{c}}+0.02$ & -0.12 & +0.06 & -0.0022 \\
\hline \hline Total models & $\pm \mathbf{0 . 1 7}$ & $\pm \mathbf{0 . 2 7}$ & $\pm \mathbf{0 . 0 0 3 1}$ \\
\hline \hline $\mathrm{BR}\left(\mathrm{b} \rightarrow \ell^{-}\right)+0.32 \%$ & -0.01 & +0.11 & +0.0011 \\
\hline $\mathrm{BR}\left(\mathrm{b} \rightarrow \mathrm{c} \rightarrow \ell^{+}\right)+0.47 \%$ & -0.03 & -0.06 & -0.0024 \\
\hline $\mathrm{BR}\left(\mathrm{b} \rightarrow \overline{\mathrm{c}} \rightarrow \ell^{-}\right)+0.50 \%$ & -0.01 & +0.17 & +0.0017 \\
\hline $\mathrm{BR}\left(\mathrm{b} \rightarrow \tau \rightarrow \ell^{-}\right)+0.20 \%$ & +0.00 & +0.07 & +0.0007 \\
\hline $\mathrm{BR}\left(\mathrm{c} \rightarrow \ell^{+}\right)+0.50 \%$ & +0.05 & -0.16 & +0.0000 \\
\hline \hline Total branching ratios & $\pm \mathbf{0 . 0 6}$ & $\pm \mathbf{0 . 2 7}$ & $\pm \mathbf{0 . 0 0 3 2}$ \\
\hline \hline Electron background increase & +0.02 & +0.04 & -0.0003 \\
\hline Muon background increase & +0.05 & +0.12 & -0.0005 \\
\hline Conversion fraction increase & +0.04 & +0.09 & -0.0004 \\
\hline Decay fraction increase & +0.02 & +0.04 & -0.0002 \\
\hline Source dependence & +0.03 & +0.09 & +0.0007 \\
\hline Tracking Resolution & -0.06 & +0.01 & +0.0011 \\
\hline Input corrections & +0.03 & -0.02 & -0.0007 \\
\hline \hline Total detector effects & $\pm \mathbf{0 . 1 1}$ & $\pm \mathbf{0 . 1 9}$ & $\pm \mathbf{0 . 0 0 1 8}$ \\
\hline \hline$\theta$ dependence & +0.06 & -0.11 & +0.0007 \\
\hline Monte Carlo statistics & +0.07 & +0.06 & +0.0027 \\
\hline Time dependent mixing & +0.04 & +0.04 & +0.0000 \\
\hline Background asymmetry $+0.5 \%$ & +0.01 & +0.27 & +0.0000 \\
\hline \hline Total other systematics & $\pm \mathbf{0 . 0 9}$ & $\pm \mathbf{0 . 3 0}$ & $\pm \mathbf{0 . 0 0 2 7}$ \\
\hline \hline
\end{tabular}

Table 10: Sources of systematic errors for the on-peak measurement. 


\begin{tabular}{lcccc}
\hline & \multicolumn{2}{c}{ peak-2 } & \multicolumn{2}{c}{ peak +2} \\
\hline Fitted value & $A_{\mathrm{FB}}^{\mathrm{b}}(\%)$ & $A_{\mathrm{FB}}^{\mathrm{c}}(\%)$ & $A_{\mathrm{FB}}^{\mathrm{b}}(\%)$ & $A_{\mathrm{FB}}^{\mathrm{c}}(\%)$ \\
\hline Statistical error & 5.55 & -7.52 & 11.70 & 14.08 \\
\hline Systematic error & \pm 2.39 & \pm 3.38 & \pm 2.02 & \pm 2.80 \\
\hline
\end{tabular}

Sources of systematic errors

\begin{tabular}{lcccc}
\hline $\mathrm{b} \rightarrow \ell^{-}(\mathrm{ACCMM}$ to ISGWW**) & +0.07 & +0.07 & +0.05 & +0.32 \\
\hline $\mathrm{c} \rightarrow \ell^{+}(\mathrm{ACCMM}$ to ACCMM1) & -0.05 & +0.01 & +0.13 & -0.15 \\
\hline$\left\langle x_{E}\right\rangle_{\mathrm{b}}-0.02$ & -0.15 & -0.19 & -0.06 & +0.09 \\
\hline$\left\langle x_{E}\right\rangle_{\mathrm{c}}+0.02$ & +0.03 & -0.10 & -0.21 & +0.25 \\
\hline \hline Total models & $\pm \mathbf{0 . 1 7}$ & $\pm \mathbf{0 . 2 3}$ & $\pm \mathbf{0 . 2 6}$ & $\pm \mathbf{0 . 4 4}$ \\
\hline \hline $\mathrm{BR}\left(\mathrm{b} \rightarrow \ell^{-}\right)+0.32 \%$ & +0.03 & -0.01 & -0.03 & +0.18 \\
\hline $\mathrm{BR}\left(\mathrm{b} \rightarrow \mathrm{c} \rightarrow \ell^{+}\right)+0.47 \%$ & -0.09 & -0.13 & -0.02 & -0.05 \\
\hline $\mathrm{BR}\left(\mathrm{b} \rightarrow \overline{\mathbf{c}} \rightarrow \ell^{-}\right)+0.50 \%$ & +0.05 & -0.02 & -0.04 & +0.28 \\
\hline $\mathrm{BR}\left(\mathrm{b} \rightarrow \tau \rightarrow \ell^{-}\right)+0.20 \%$ & +0.02 & -0.01 & -0.02 & +0.11 \\
\hline $\mathrm{BR}\left(\mathrm{c} \rightarrow \ell^{+}\right)+0.50 \%$ & +0.02 & +0.12 & +0.08 & -0.32 \\
\hline \hline Total branching ratios & $\pm \mathbf{0 . 1 1}$ & $\pm \mathbf{0 . 1 8}$ & $\pm \mathbf{0 . 1 0}$ & $\pm \mathbf{0 . 4 8}$ \\
\hline \hline Electron background increase & +0.01 & -0.03 & +0.03 & +0.06 \\
\hline Muon background increase & +0.02 & -0.11 & +0.07 & +0.26 \\
\hline Conversion fraction increase & +0.04 & -0.05 & +0.07 & +0.17 \\
\hline Decay fraction increase & +0.01 & -0.03 & +0.03 & +0.08 \\
\hline Source dependence & +0.03 & +0.08 & +0.04 & +0.17 \\
\hline Tracking resolution & +0.01 & -0.14 & -0.06 & +0.03 \\
\hline Input corrections & -0.04 & -0.05 & +0.02 & +0.06 \\
\hline \hline Total detector effects & $\pm \mathbf{0 . 1 2}$ & $\pm \mathbf{0 . 3 0}$ & $\pm \mathbf{0 . 1 3}$ & $\pm \mathbf{0 . 3 9}$ \\
\hline \hline$\theta$ dependence & +0.14 & +0.30 & +0.09 & -0.19 \\
\hline Monte Carlo statistics & +0.12 & +0.14 & +0.08 & +0.28 \\
\hline Time dependent mixing & +0.04 & +0.04 & +0.04 & +0.04 \\
\hline \hline Background asymmetry $+0.5 \%$ & +0.00 & +0.27 & +0.01 & +0.28 \\
\hline Total other systematics & $\pm \mathbf{0 . 1 6}$ & $\pm \mathbf{0 . 3 6}$ & $\pm \mathbf{0 . 1 2}$ & $\pm \mathbf{0 . 4 3}$ \\
\hline \hline
\end{tabular}

Table 11: Sources of systematic errors for the two off-peak measurements. 


\section{Conclusion}

Using all data collected by OPAL between 1990 and 1994, the b and c quark forward-backward asymmetries have been measured at three centre-of-mass energy points on and around the $Z^{0}$ peak, and were found to be:

$$
\begin{array}{lll}
A_{\mathrm{FB}}^{\mathrm{b}}=(5.5 \pm 2.4 \pm 0.3) \% & A_{\mathrm{FB}}^{\mathrm{c}}=(-7.5 \pm 3.4 \pm 0.6) \% & \text { at }\langle\sqrt{s}\rangle=89.52 \mathrm{GeV}, \\
A_{\mathrm{FB}}^{\mathrm{b}}=(9.06 \pm 0.51 \pm 0.23) \% & A_{\mathrm{FB}}^{\mathrm{c}}=(6.00 \pm 0.67 \pm 0.52) \% & \text { at }\langle\sqrt{s}\rangle=91.24 \mathrm{GeV}, \\
A_{\mathrm{FB}}^{\mathrm{b}}=(11.7 \pm 2.0 \pm 0.3) \% & A_{\mathrm{FB}}^{\mathrm{c}}=(14.1 \pm 2.8 \pm 0.9) \% & \text { at }\langle\sqrt{s}\rangle=92.94 \mathrm{GeV}
\end{array}
$$

The average $b$ mixing has been extracted simultaneously with the asymmetries and was measured to be:

$$
\bar{\chi}=0.1107 \pm 0.0062 \pm 0.0055 .
$$

In each case, the first quoted error is statistical while the second is systematic.

These measurements of $A_{\mathrm{FB}}^{\mathrm{b}}$ and $A_{\mathrm{FB}}^{\mathrm{c}}$ supersede the previous measurements with leptons by OPAL [5]. They are compatible with other measurements from LEP experiments [3-7,34] and with the Standard Model predictions (figures 8 and 9 ).

Assuming the Standard Model and $m_{\mathrm{H}}=300 \mathrm{GeV} / c^{2}$, the measurements of $A_{\mathrm{FB}}^{\mathrm{b}}$ and $A_{\mathrm{FB}}^{\mathrm{c}}$ at $\langle\sqrt{s}\rangle=$ $91.24 \mathrm{GeV}$ lead to the value of $m_{\mathrm{top}}$ :

$$
m_{\mathrm{top}}=179 \pm 23 \text { (stat) } \pm 12(\text { syst }) \pm 22\left(m_{\mathrm{H}}\right) \mathrm{GeV} / c^{2}
$$

where the last error corresponds to a variation of $m_{\mathrm{H}}$ between 60 and $1000 \mathrm{GeV} / \mathrm{c}^{2}$. This is in $\operatorname{good}$ agreement with the combined CDF and D0 average of: $m_{\text {top }}=180 \pm 12 \mathrm{GeV} / c^{2}[35]$.

\section{Acknowledgements}

It is a pleasure to thank the SL Division for the efficient operation of the LEP accelerator, the precise information on the absolute energy, and their continuing close cooperation with our experimental group. In addition to the support staff at our own institutions we are pleased to acknowledge the Department of Energy, USA,

National Science Foundation, USA,

Particle Physics and Astronomy Research Council, UK,

Natural Sciences and Engineering Research Council, Canada,

Fussefeld Foundation,

Israel Ministry of Science,

Israel Science Foundation, administered by the Israel Academy of Science and Humanities,

Minerva Gesellschaft,

Japanese Ministry of Education, Science and Culture (the Monbusho) and a grant under the Mon-

busho International Science Research Program,

German Israeli Bi-national Science Foundation (GIF),

Direction des Sciences de la Matière du Commissariat à l'Energie Atomique, France,

Bundesministerium für Forschung und Technologie, Germany,

National Research Council of Canada,

Hungarian Foundation for Scientific Research, OTKA T-016660, and OTKA F-015089. 


\section{References}

[1] The LEP Collaborations ALEPH, DELPHI, L3, OPAL and the LEP Electroweak Working Group, A combination of Preliminary LEP Electroweak Measurements and Constraints on the Standard Model, CERN-PPE/95-172.

[2] P. Franzini, Physics Reports 173 (1989) 1.

[3] ALEPH Collaboration, D. Buskulic et al., Z. Phys. C62 (1994) 179.

[4] DELPHI Collaboration, P. Abreu et al., Z. Phys. C65 (1995) 569;

L3 Collaboration, M. Acciari et al., Phys. Lett. B335 (1994) 542;

ALEPH Collaboration, D. Buskulic et al., Phys. Lett. B335 (1994) 99.

[5] OPAL Collaboration, P. Acton et al., Z. Phys. C60 (1993) 19.

[6] OPAL Collaboration, R. Akers et al., Z. Phys. C60 (1993) 601.

[7] OPAL Collaboration, R. Akers et al., Z. Phys. C67 (1995) 365.

[8] OPAL Collaboration, K. Ahmet et al., Nucl. Instrum. Meth. A305 (1991) 275.

P. P. Allport et al., Nucl. Inst. and Meth. A324 (1993) 34;

P. P. Allport et al., Nucl. Inst. and Meth. A346 (1994) 479.

[9] OPAL Collaboration, G. Alexander et al., Z. Phys. C52 (1991) 175.

[10] OPAL Collaboration, R. Akers et al., Z. Phys. C63 (1994) 197.

[11] JADE Collaboration, Z. Phys. C33 (1986) 23.

[12] T. Sjöstrand, Comp. Phys. Comm. 39 (1986) 347;

T. Sjöstrand, JETSET 7.3: physics and manual, CERN-TH/92-6488.

[13] OPAL Collaboration, P. Acton et al., Z. Phys. C58 (1993) 387.

[14] J. Allison et al., Nucl. Instrum. Meth. A317 (1992) 47.

[15] C. Peterson et al., Phys. Rev. D27 (1983) 105.

[16] G. Altarelli et al. Nucl. Phys. B208 (1982) 365.

[17] CLEO Collaboration, S. Henderson et al., Phys. Rev. D 45 (1992) 2212.

[18] DELCO Collaboration, W. Bacino et al., Phys. Rev. Lett. 43 (1979) 1073.

[19] MARK III Collaboration, R.M. Baltrusaitis et al., Phys. Rev. Lett. 54 (1985) 1976.

[20] OPAL Collaboration, R. Akers et al., Phys. Lett. B327 (1994) 411.

[21] D.E. Rumelhart and J.L. McClelland, Parallel Distributed Processing, MIT Press (1986).

[22] OPAL Collaboration, R. Akers et al., Z. Phys. C65 (1995) 17.

[23] OPAL Collaboration, R. Akers et al., Z. Phys. C60 (1993) 199.

[24] OPAL Collaboration, R. Akers et al., Z. Phys. C66 (1995) 555.

[25] OPAL Collaboration, R. Akers et al., Phys. Lett. B352 (1995) 176.

[26] D. Bardin et al., CERN-TH 6443/92, May 1992. 
[27] The LEP Collaborations ALEPH, DELPHI, L3, OPAL and the LEP Heavy Flavour Electroweak Working Group, Combination of Heavy Flavour Electroweak Measurements at LEP, CERNPPE/96-xxx.

[28] DELPHI Collaboration, P. Abreu et al., Z. Phys. C57 (1993) 181;

L3 Collaboration, B. Adeva et al., Phys. Lett. B261 (1991) 177;

DELPHI Collaboration, P. Abrew et al., Z. Phys. C59 (1993) 533.

[29] N. Isgur, D. Scora, B. Grinstein and M. Wise, Phys. Rev. D39 (1989) 799.

[30] CLEO Collaboration, D. Bortoletto et al., Phys. Rev. D45 (1992) 21.

[31] DELPHI Collaboration, Z. Phys. C66 (1995) 323.

[32] ARGUS Collaboration, H. Albrecht et al., Phys. Lett. B278 (1992) 202.

[33] ALEPH Collaboration, D. Buskulic et al., Phys. Lett. B298 (1993) 479.

[34] Particle Data Group, Phys. Rev. D50 (1994) 1.

[35] CDF Collaboration, F. Abe et al., Phys. Rev. Lett. 74 (1995) 2626.

D0 Collaboration, S. Abachi et al., Phys. Rev. Lett. 74 (1995) 2632. 

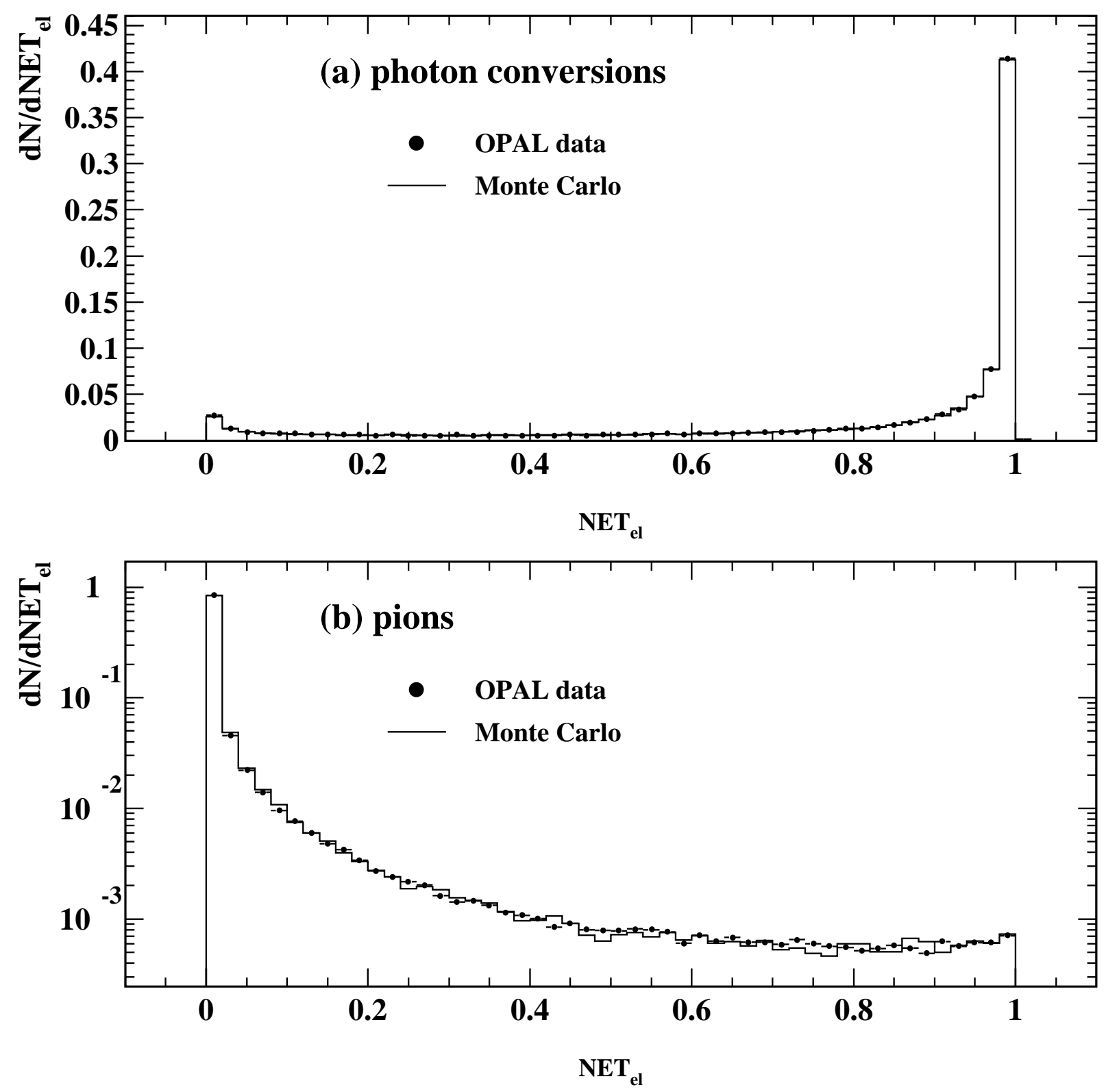

Figure 1: Comparison of the normalized $\mathrm{NET}_{\mathrm{el}}$ distribution (a) for electrons tagged as conversions and (b) for pions from $\mathrm{K}^{0}$ decays, in data (points with error bars) and in the simulation (histogram). 


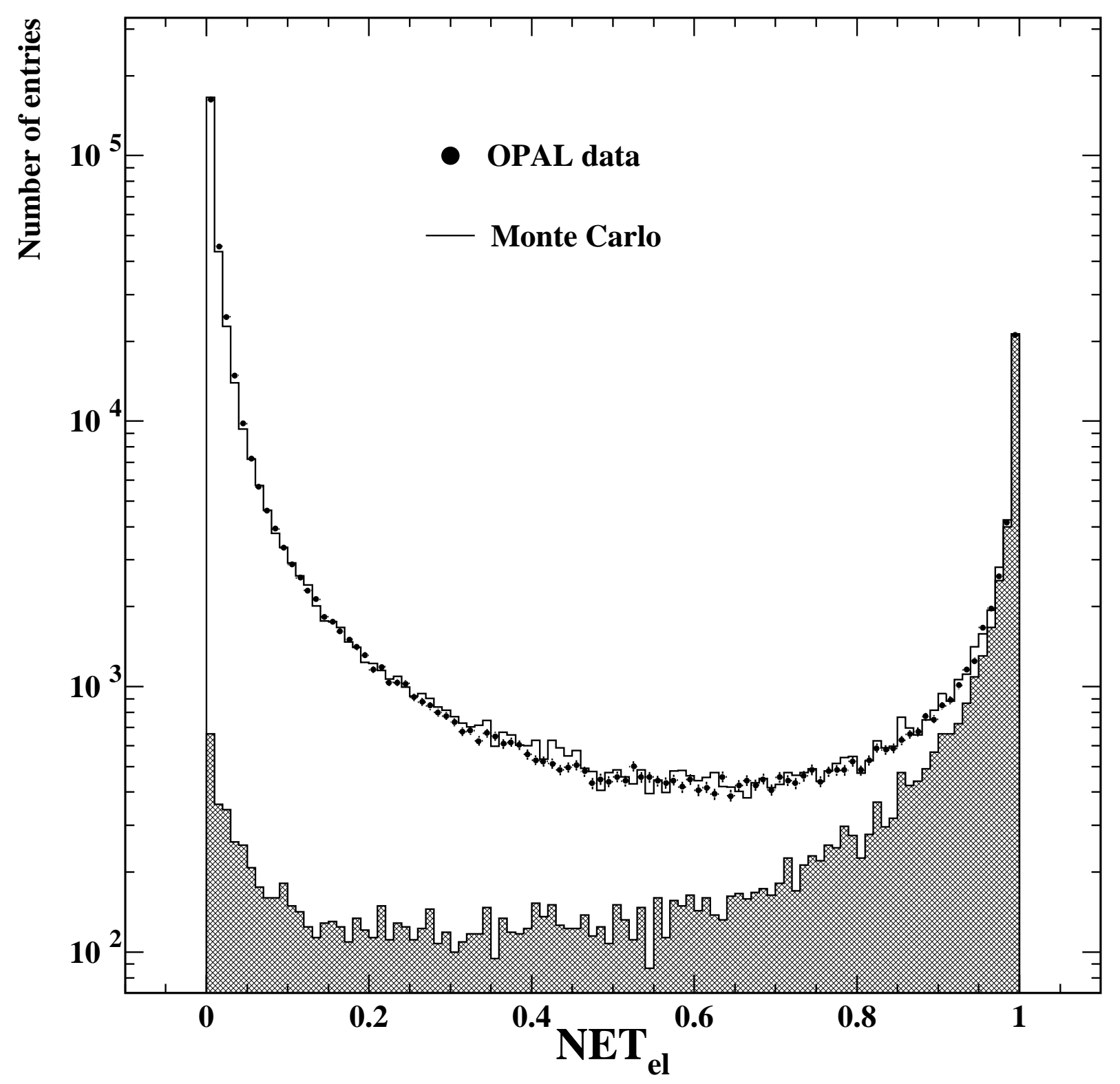

Figure 2: Distributions of the output of the neural network NET $_{\text {el }}$ used in the electron selection, for data (points) and Monte Carlo (histogram), normalized to the same number of entries, for all tracks with $p>2 \mathrm{GeV} / c$. The distribution for all MC electrons (shaded area) is clearly peaked at 1 . 

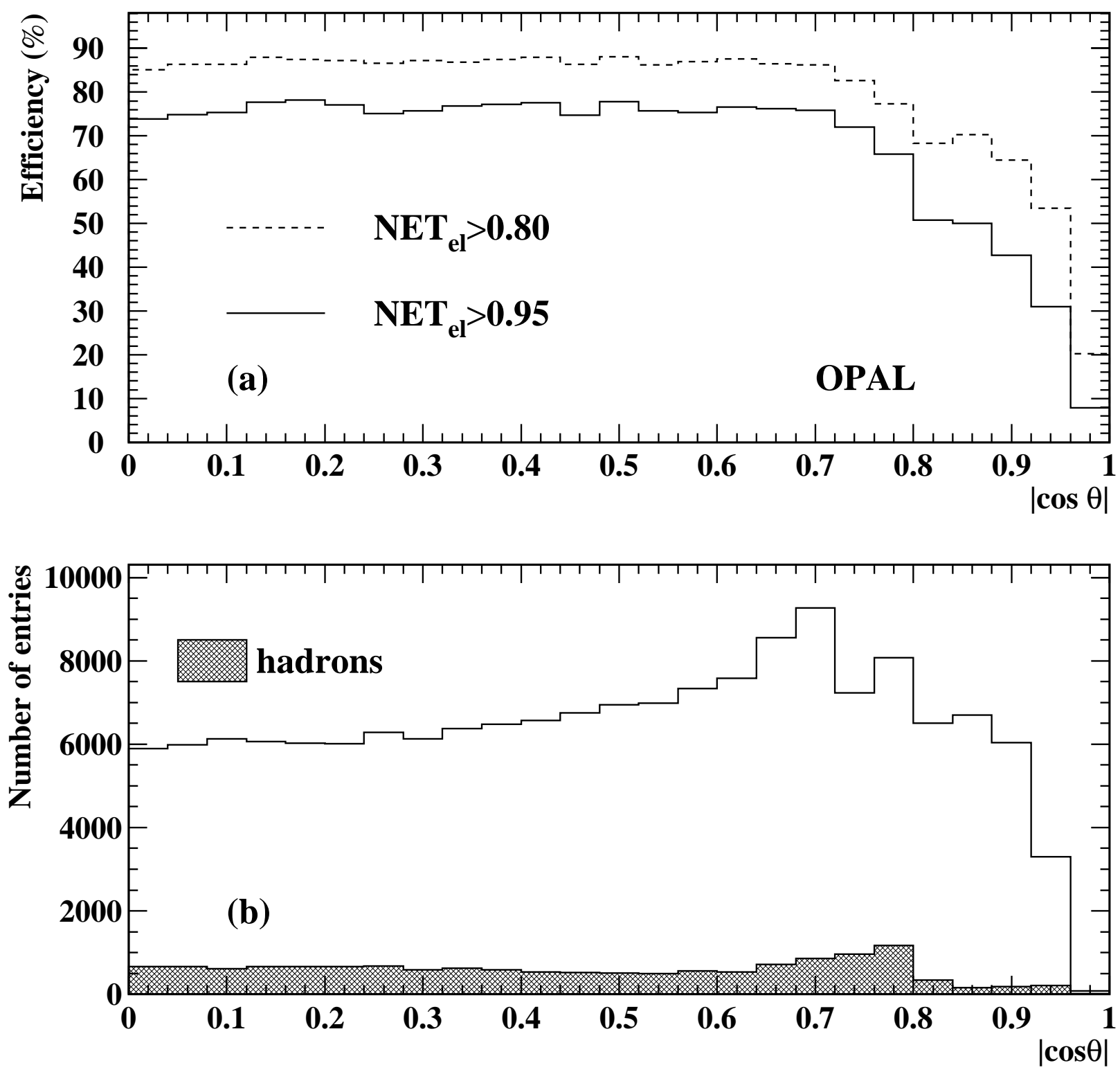

Figure 3: (a) Efficiency of the electron selection for all Monte Carlo prompt electrons with $p>2 \mathrm{GeV} / c$ as a function of the polar angle $\theta,(\mathrm{b})|\cos \theta|$ distribution of candidate electrons in the data. The shaded area represents the hadronic background contribution as predicted by the simulation. 

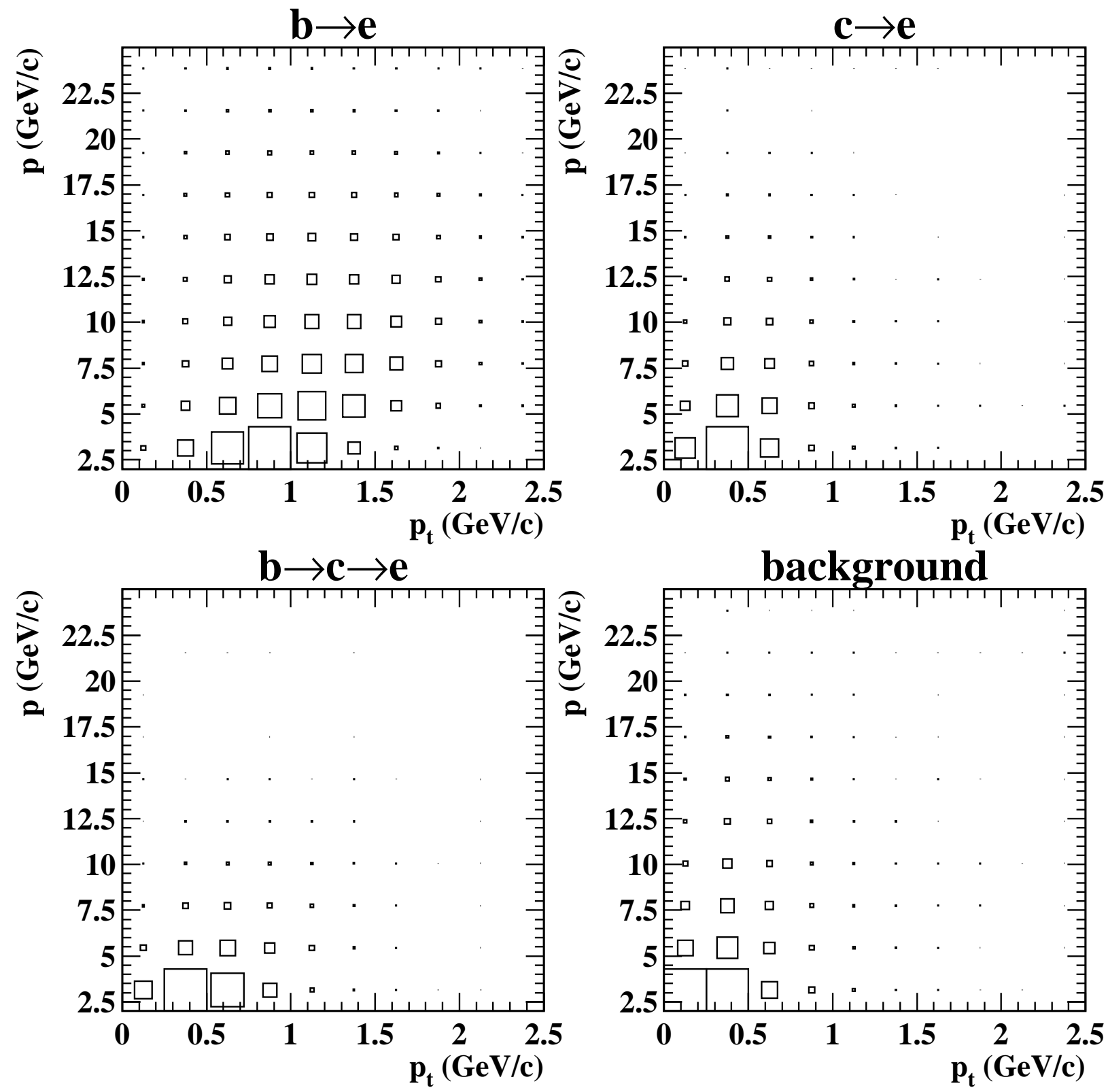

Figure 4: Distributions of candidate electrons from different sources in the $\left(p, p_{t}\right)$ plane taken from the simulation. The area of each square is proportional to the number of events in that region. 

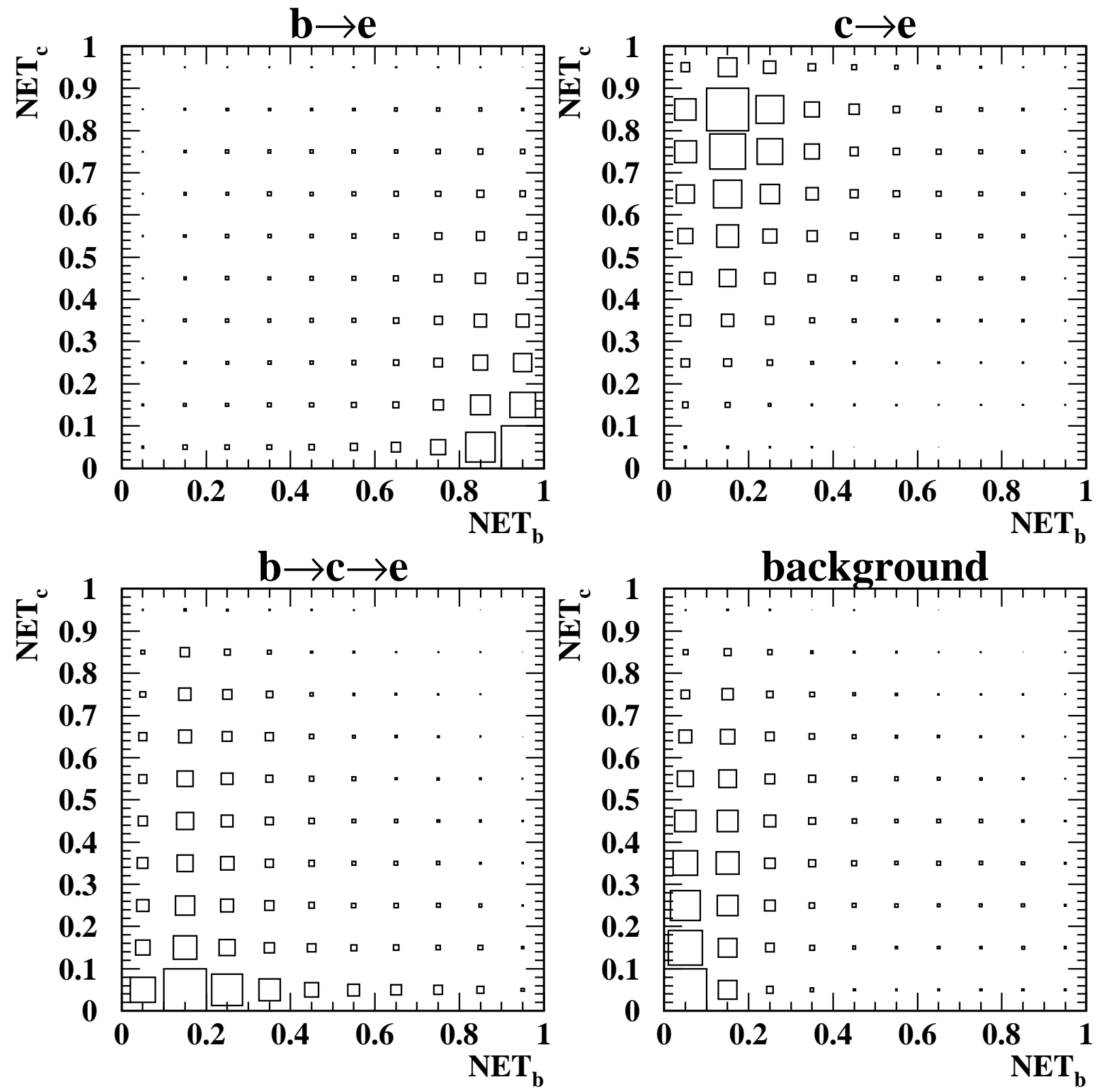

Figure 5: Distributions of candidate electrons from different sources in the $\left(\mathrm{NET}_{\mathrm{b}}, \mathrm{NET}_{\mathrm{c}}\right)$ plane taken from the simulation. The area of each square is proportional to the number of events in that region. 

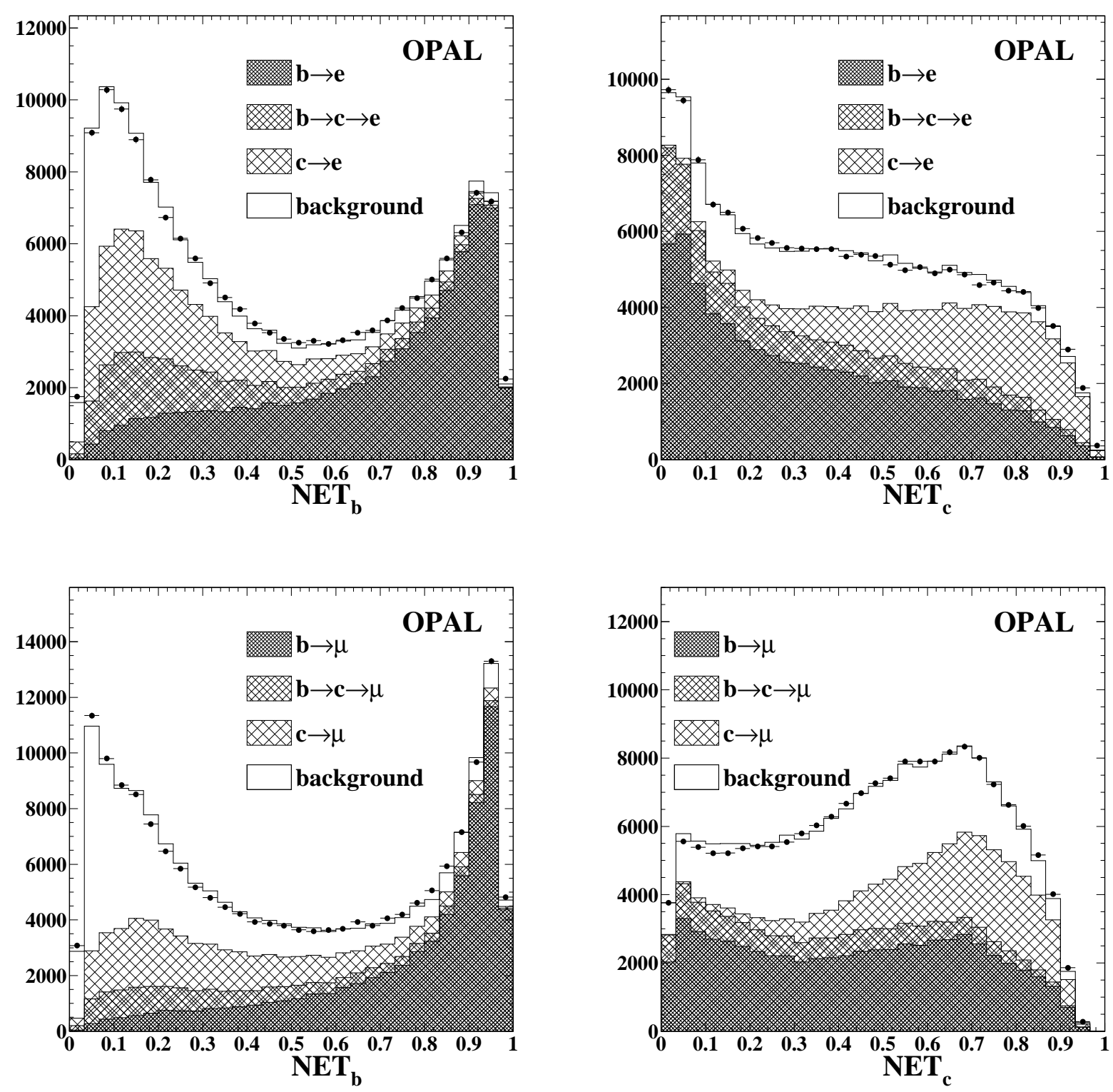

Figure 6: Distributions of the separating variables $\mathrm{NET}_{\mathrm{b}}$ and $\mathrm{NET}_{\mathrm{c}}$ for electrons (top) and muons (bottom). The predicted Monte Carlo distributions (histogram) are superimposed on the distributions measured in the data (points). The contributions of candidate leptons from different sources are also indicated. 

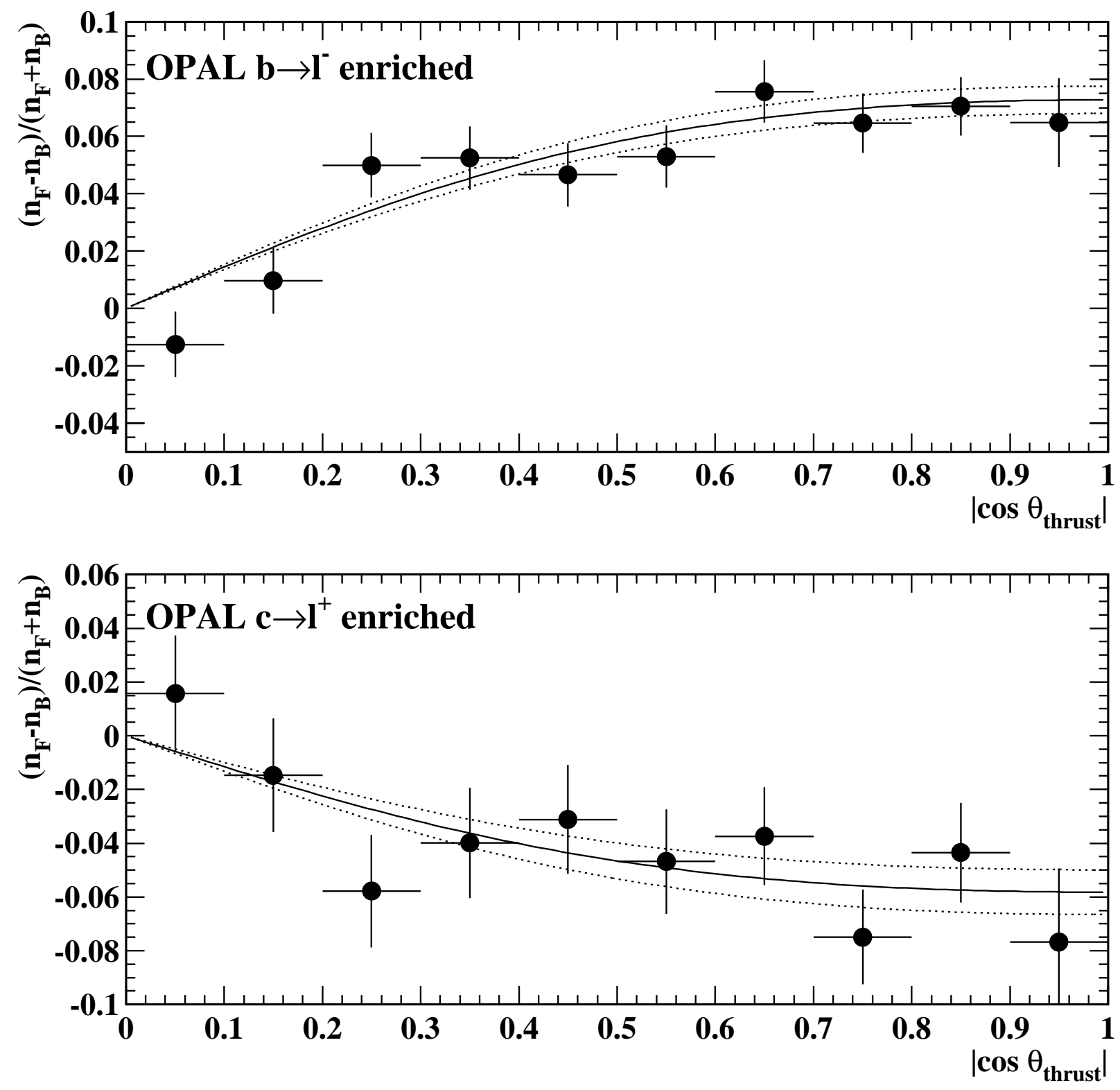

Figure 7: The ratio $\left(n_{F}-n_{B}\right) /\left(n_{F}+n_{B}\right)$ as a function of $|y|=\left|\cos \theta_{\text {thrust }}\right|$ in the $\mathrm{b} \rightarrow \ell^{-}$and $\mathrm{c} \rightarrow \ell^{+}$ enriched samples. The curves are fitted to this ratio with the form $8 A_{\mathrm{FB}}|y| / 3\left(1+y^{2}\right)$. The solid curves correspond to the fitted values of $A_{\mathrm{FB}}^{\mathrm{b}}$ and $A_{\mathrm{FB}}^{\mathrm{c}}$ given in table 7 and the dotted curves to one standard deviation from the fitted value. 

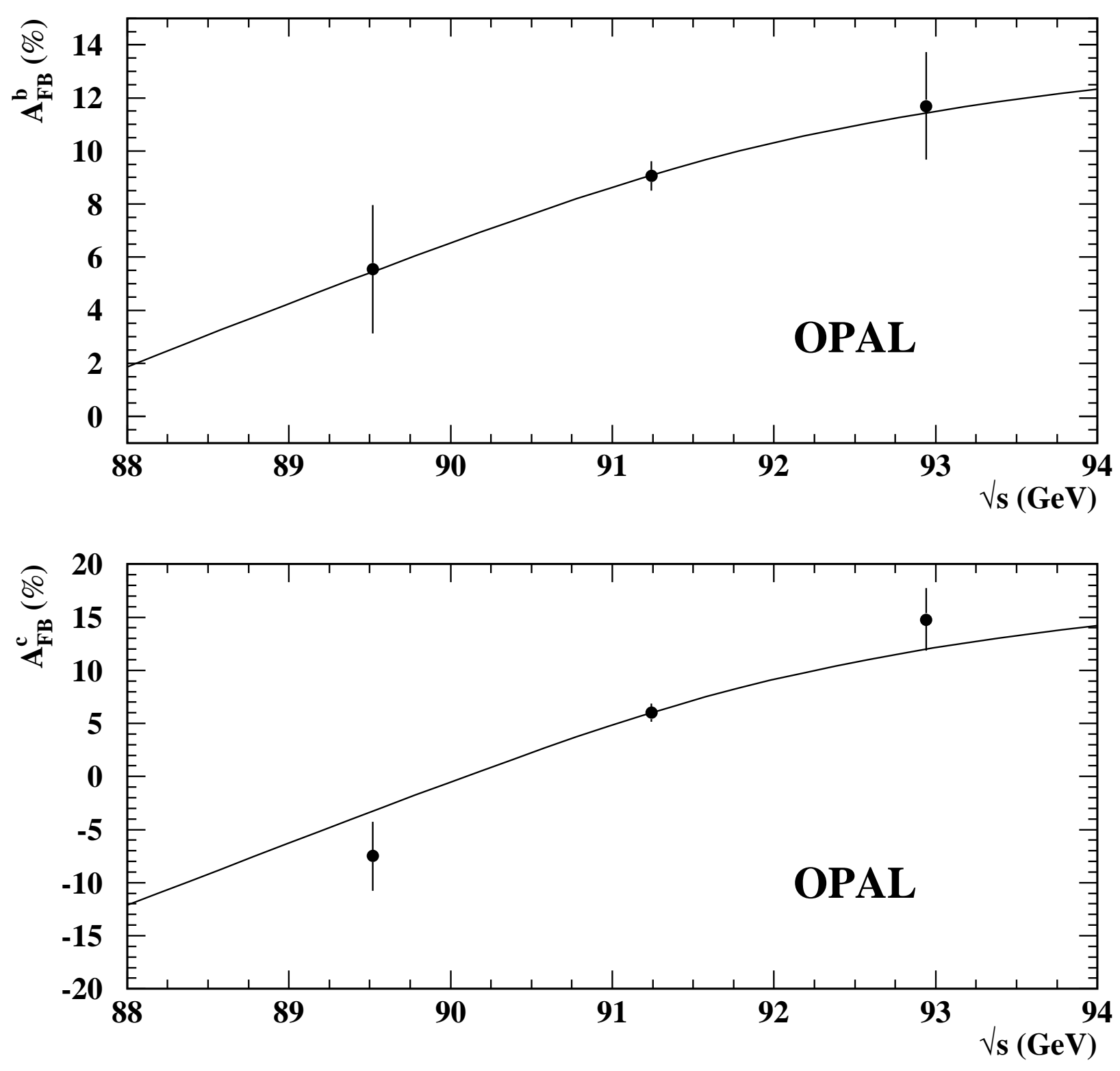

Figure 8: Measurements of $A_{\mathrm{FB}}^{\mathrm{b}}$ and $A_{\mathrm{FB}}^{\mathrm{c}}$ as a function of the $\mathrm{e}^{+} \mathrm{e}^{-}$centre-of-mass energy $\sqrt{s}$. The curve corresponds to the Standard Model prediction with $m_{\mathrm{Z}}=91.187 \mathrm{GeV} / c^{2}, m_{\mathrm{top}}=180 \mathrm{GeV} / c^{2}$, $m_{\mathrm{H}}=300 \mathrm{GeV} / c^{2}$ and $\alpha_{s}=0.125$. 


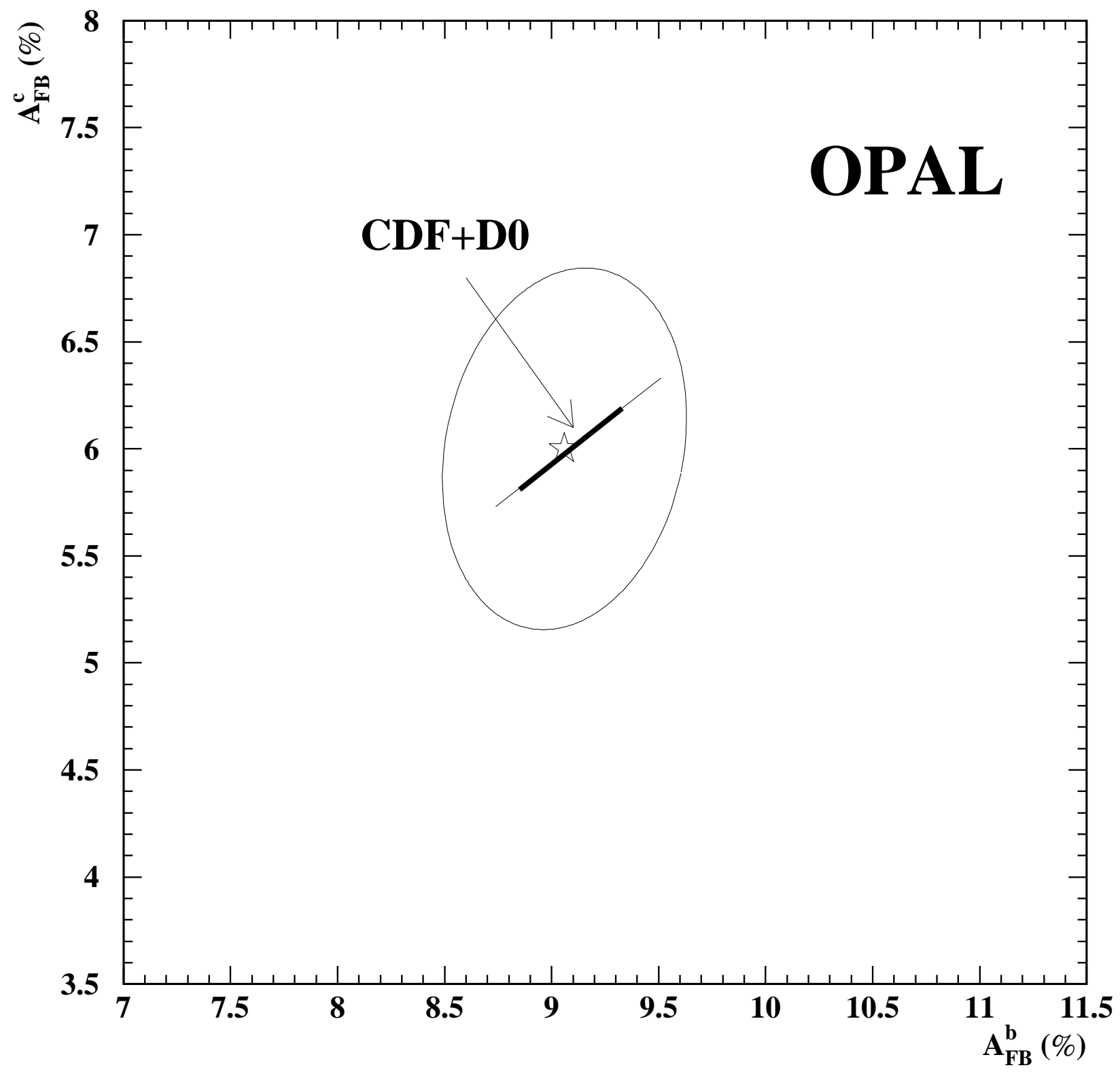

Figure 9: One standard deviation contour (39\% probability content) for $A_{\mathrm{FB}}^{\mathrm{b}}$ and $A_{\mathrm{FB}}^{\mathrm{c}}$. The thick line is the Standard Model prediction for $m_{\mathrm{Z}}=91.187 \mathrm{GeV} / c^{2}, m_{\mathrm{H}}=300 \mathrm{GeV} / c^{2}, \alpha_{s}=0.125$ and $m_{\text {top }}=$ $180 \pm 12 \mathrm{GeV} / c^{2}$. The thin line is obtained for $m_{\mathrm{top}}=180 \mathrm{GeV} / c^{2}$ and $m_{\mathrm{H}}$ varying between 60 and $1000 \mathrm{GeV} / c^{2}$. 